OPEN ACCESS

Edited by:

Peter M. Van Endert,

Institut National de la Santé et de la Recherche Médicale (INSERM),

France

Reviewed by:

Pedro A. Reche,

Complutense University of Madrid,

Spain

Erika Margaret Scholz Valero, Universitat Autònoma de Barcelona,

Spain

${ }^{*}$ Correspondence: Morten Nielsen morni@dtu.dk

${ }^{\dagger}$ These authors have contributed equally to this work

Specialty section:

This article was submitted to Antigen Presenting Cell Biology, a section of the journal

Frontiers in Immunology

Received: 14 December 2021 Accepted: 10 January 2022 Published: 26 January 2022

Citation:

Kaabinejadian S, Barra C, Alvarez B, Yari $\mathrm{H}$, Hildebrand $\mathrm{WH}$ and Nielsen $M$ (2022) Accurate MHC Motif Deconvolution of Immunopeptidomics

Data Reveals a Significant Contribution of DRB3, 4 and 5 to the

Total DR Immunopeptidome.

Front. Immunol. 13:835454. doi: 10.3389/fimmu.2022.835454

\section{Accurate MHC Motif Deconvolution of Immunopeptidomics Data Reveals a Significant Contribution of DRB3, 4 and 5 to the Total DR Immunopeptidome}

\author{
Saghar Kaabinejadian ${ }^{1,2+}$, Carolina Barra ${ }^{3+}$, Bruno Alvarez ${ }^{4}$, Hooman Yari ${ }^{2}$, \\ William H. Hildebrand ${ }^{2}$ and Morten Nielsen ${ }^{3,4 *}$ \\ 1 Pure MHC, LLC., Oklahoma City, OK, United States, 2 Department of Microbiology and Immunology, University of \\ Oklahoma Health Sciences Center, Oklahoma City, OK, United States, ${ }^{3}$ Department of Health Technology, Technical \\ University of Denmark, Lyngby, Denmark, ${ }^{4}$ Instituto de Investigaciones Biotecnológicas, Universidad Nacional de San Martín, \\ Buenos Aires, Argentina
}

Mass spectrometry (MS) based immunopeptidomics is used in several biomedical applications including neo-epitope discovery in oncology, next-generation vaccine development and protein-drug immunogenicity assessment. Immunopeptidome data are highly complex given the expression of multiple HLA alleles on the cell membrane and presence of co-immunoprecipitated contaminants. The absence of tools that deal with these challenges effectively and guide the analysis and interpretation of this complex type of data is currently a major bottleneck for the large-scale application of this technique. To resolve this, we here present the MHCMotifDecon that benefits from state-of-the-art HLA class-I and class-II predictions to accurately deconvolute immunopeptidome datasets and assign individual ligands to the most likely $H\llcorner A$ molecule, allowing to identify and characterize HLA binding motifs while discarding co-purified contaminants. We have benchmarked the tool against other state-of-the-art methods and illustrated its application on experimental datasets for HLA-DR demonstrating a previously underappreciated role for HLA-DRB3/4/5 molecules in defining HLA class II immune repertoires. With its ease of use, MHCMotifDecon can efficiently guide interpretation of immunopeptidome datasets, serving the discovery of novel T cell targets. MHCMotifDecon is available at https://services.healthtech.dtu.dk/service.php?MHCMotifDecon-1.0.

Keywords: MHCMotifDecon, MHC motif deconvolution, immunopeptidome, DRB3/4/5, mass spectrometry

\section{INTRODUCTION}

Mass spectrometry (MS) based immunopeptidomics is becoming increasingly relevant for several biomedical applications including cancer neoantigen discovery, vaccine development and protein drug deimmunization pipelines (1-4). The broad interest in MHC-derived MS relies on its ability to discover MHC presented peptides in real scenarios, such as biomarker discovery in cancer, 
autoimmune and infectious disease, and therefore identifying peptides that might induce $\mathrm{T}$ cell responses. Additionally, thousands of peptides derived from the self-proteins can be collected in a single assay which can be directly loaded into bioinformatic motif identification and machine learning pipelines to help uncovering the rules governing the processing and presentation of peptides in a biological context. Furthermore, ongoing refinements of the MS experimental workflows, such as de novo peptide annotation, or peptide quantification hold great promise in expanding the usage of the technique.

Despite the great potential of MS immunopeptidomics data, several inherent challenges curb the broad benefit of the technique. A major challenge being assigning the accurate MHC allele to each presented MS-derived peptide, a process termed motif deconvolution. To tackle this concern, several experimental approaches that artificially engineer cells to only express one individual HLA have been performed both for MHC class I $(5,6)$, and MHC class II (7). Although this laborious experimental setup has proved extremely useful to understand the rules of HLA presentation and their motifs, it lacks the biological relevance, limiting its application in other scenarios and experimental settings that involve patient samples and biospecimens.

As an alternative to experimental approaches, unsupervised bioinformatic methods have been proposed to deconvolute the peptide specificities present in complex immunopeptidomes, such as GibbsCluster $(8,9)$ or MixMHCp (10). These pioneering tools have proved to be highly useful for MS data analysis (11-14). However, they also show intrinsic limitations such as the need for manual inspection associating those motifs to known allele specificities present in the sample and assigning peptides to MHC molecules with overlapping motifs. Another challenge for these methods is dealing with proper deconvolution of MHC molecules with limited peptide repertoire such as HLAC. Furthermore, in MHC class II, the expression of variable alpha and beta genes forming the HLA-DQ and HLA-DP heterodimers, plus the expression of HLA-DRB1 and its associated HLA-DRB3, 4 and 5 alleles, make up to twelve potential HLA class II specificities, disputing the capacity of these methods to learn from this complex data.

Recently, Alvarez et al. proposed the NNAlign_MA framework that combines binding affinity and MS data to learn and deconvolute the peptide specificity associated to MS immunopeptidome samples on the fly, while training a neural network to predict MHC presentation (15). This method showed a high deconvolution accuracy, and this core algorithm has been already introduced into the state-of-the-art predictors NetMHCpan-4.1 and NetMHCIIpan-4.0 (16).

Here, we build on these achievements to develop MHCMotifDecon, a user-friendly supervised tool, that benefits from the prior knowledge of NetMHCpan and NetMHCIIpan to perform MHC motif deconvolution of both class I and class II MS immunopeptidomes, assigning peptide sequences to their most likely HLA restriction. The output from the tool includes HLA motifs, length distributions, peptide counts, and a trash cluster to discard non-specific or contaminating coimmunoprecipitated peptides. To show its power in analyzing immunopeptidomics data, the tool is benchmarked against other state-of-the-art publicly available motif deconvolution methods on artificial MS datasets.

To further demonstrate the power of MHCMotifDecon, we generated and motif deconvoluted novel HLA-DR immunopeptidome datasets. Considering that the DR immunopeptidome of different antigen presenting cells is naturally a complex mixture of peptides presented by HLADRB1 (primary DRB) and DRB3, 4 and 5 (secondary DRB) molecules, besides the distinct roles these molecules play in disease susceptibility or protection, the deconvolution of the peptide repertoire of DRB1 molecules from the DRB3, 4 and 5 is essential for unraveling the function of class II HLA-DR in the course of autoimmune disorder progression and treatment. However, due to their strong linkage disequilibrium (LD) with the accompanying DRB1 alleles, the contribution of the secondary DRB molecules in presenting antigenic peptides to $\mathrm{T}$ cells and their role in immune activation and response has been largely overlooked.

Here, we demonstrate how MHCMotifDecon offers a simple yet highly powerful tool to address this issue, allowing for quantification of the relative contribution of DRB3, 4 and 5 molecules to the full HLA-DR peptide repertoire.

\section{RESULTS}

The proposed MHCMotifDecon method allows for HLA motif deconvolution of immunopeptidome datasets based on prediction of peptide HLA restrictions. The tool is available at https://services. healthtech.dtu.dk/service.php?MHCMotifDecon-1.0 and takes one or multiple immunopeptidome datasets as input together with information of the HLA molecules expressed in each individual cell line/sample. On the web-interface, the user can select if motif deconvolution is to be performed for class I or class II molecules, specify the length of peptides to be included in the analysis and define the threshold (cut off) to identify and discard potential MS co-purified contaminants. For further details about the tool refer to Materials and Methods. In the following sections the performance of the tool is first benchmarked against other publicly available HLA motif deconvolution methods, and next the use and value of the tool is illustrated in a series of reallife applications.

\section{Performance Evaluation}

To assess the deconvolution accuracy and value of MHCMotifDecon, the method was tested and compared to other publicly available tools on two artificial datasets (one for HLA class I and another for HLA class II) constructed combining MS peptides from experimental designs where the restriction allele was known and unique. As MHCMotifDecon is a supervised classification method, the two datasets were constructed from peptides that do not share overlap with the 
MHC data used for the training of NetMHCpan and NetMHCIIpan (for details refer to Materials and Methods).

\section{MHC Class I Deconvolution}

For MHC class I, 4400 HLA ligands of length 8-14 amino acids were randomly sampled from six single-allele datasets (HLA$A^{\star} 02: 02$, HLA- ${ }^{\star} 11: 02$, HLA-B ${ }^{\star} 13: 01$, HLA- ${ }^{\star} 49: 01$, HLA$C^{\star} 07: 02, H L A-C^{\star} 14: 03$ ) (5) and merged into one artificial dataset. HLA-A and HLA-B ligands were added in equal proportions and five times more compared to HLA-C (17), mimicking the biological setting where HLA-A and HLA-B alleles are expressed at higher levels compared to HLA-C.

Next, MHCMotifDecon, MixMHCp (18) and GibbsCluster (8) were used to deconvolute the motifs contained within this artificial MHC class I dataset of 4400 ligands covering 6 HLA molecules. The result of this deconvolution is shown in Figure $\mathbf{1}$. As an additional comparison of the deconvoluted motifs, each of the original single allele data was submitted to GibbsCluster (11) with a fixed cluster number of 1 to show the motifs found in the original MS samples (Figure 1).

While all clustering algorithms were able to separate most HLA-A and HLA-B associated peptides, the low representation of HLA-C peptides in combination with a less defined motif makes this motif more difficult to establish (Figure 1). Focusing on the identified motifs for HLA-C*07:02 and HLA-C ${ }^{\star} 14: 03$, it becomes further apparent that both MixMHCp and GibbsCluster fail to accurately separate the correct motifs for the two HLA-C molecules (Figure 1, SA panel).

The accuracy of the motif deconvolution was next quantified in terms of a confusion matrix aligning the annotated and predicted HLA restrictions for each of the 4400 peptides in the dataset (Figure 2). These results demonstrate an overall high ability of the three methods to deconvolute the motifs from HLA-A and HLA-B, but also illustrates the difficulty of the two unsupervised methods to accurately capture the motifs of the two HLA-C molecules.

To further quantify this, we next estimated, using the HLA association for each cluster as defined in Figure $\mathbf{1}$ and as indicated along the diagonal of each matrix in (Figures 2A-C), the performance of each method in terms of the Matthews correlation of each HLA/group association. These MCC values for each method and HLA are shown in Figure 2D, and confirm the earlier observation with an overall superior performance of MHCMotifDecon and a suboptimal performance of MixMHCp and GibbsClusters for identification of the HLA-C molecule motifs.

\section{MHC Class II Deconvolution}

To assess the deconvolution efficacy of MHCMotifDecon for HLA class II immunopeptidome data, the method was applied to an artificial dataset generated merging single allele datasets from four different cell lines expressing DRB1 $1^{\star} 04: 03$, DRB $1^{\star} 08: 03$, DRB $3^{*} 02: 02$ and DRB $5^{*} 01: 01$ alleles respectively (for details on the artificial dataset generation refer to Materials and Methods section). Here, the motif deconvolution was performed using the new retrained version 4.1 of NetMHCIIpan. The retraining was performed as described in Materials and Methods expanding the data to include an extended set of single allele MS data, excluding single allele data from the alleles used on the benchmark to avoid overestimation of the deconvolution accuracy. Also in this benchmark two alternative methods, GibbsCluster and MoDec were included. The result of the benchmark is shown in Figure 3,

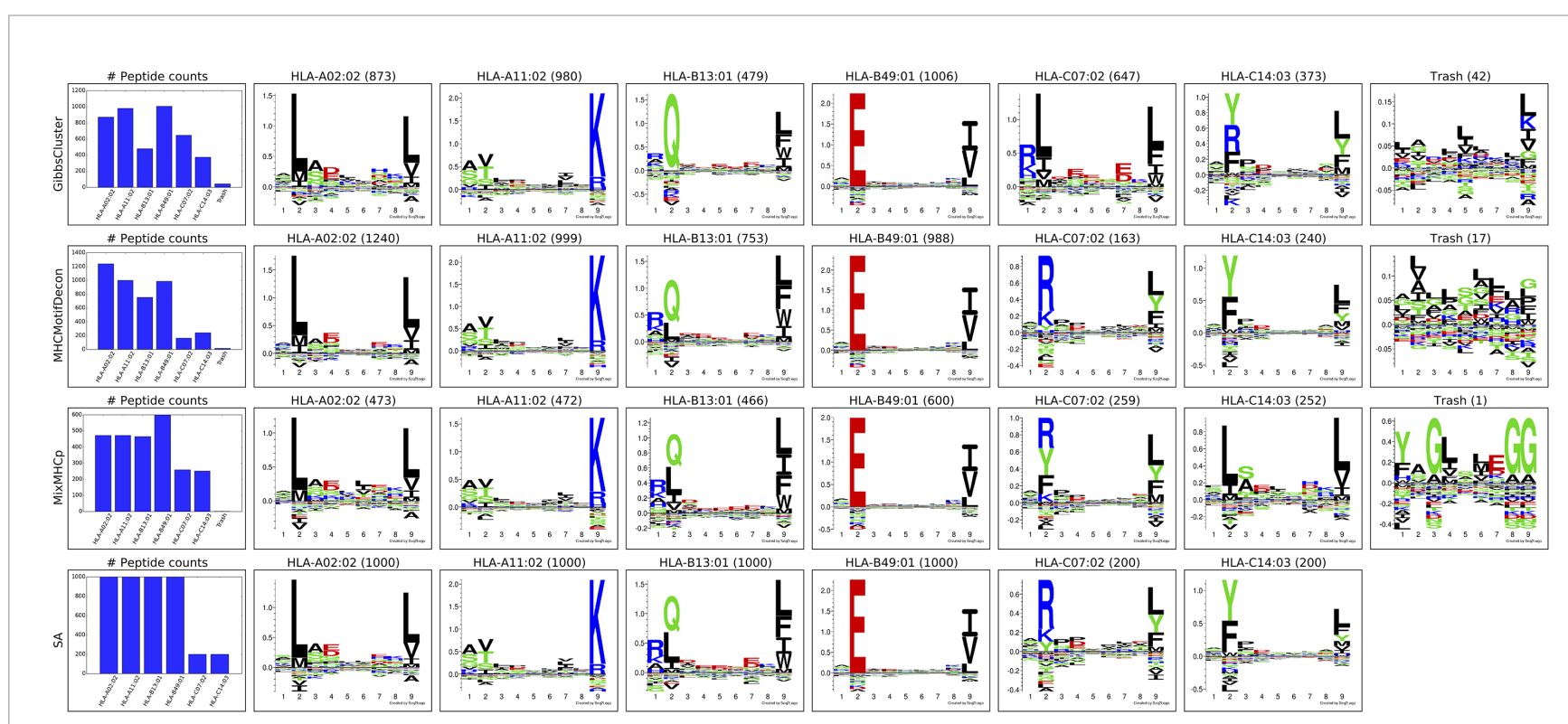

FIGURE 1 | Motif deconvolution of the artificial HLA class I dataset. GibbsCluster, MHCMotifDecon, MixMHCp deconvolution of datasets. Motifs for MixMHCp were constructed from the set of deconvoluted 9mer peptides only. The lower panel (SA) shows motifs for each individual HLA-A*02:02, HLA-A*11:02, HLA-B*13:01, HLA-B*49:01, HLA-C*07:02, HLA-C*14:03 dataset as obtained by GibbsCluster using a single cluster. 

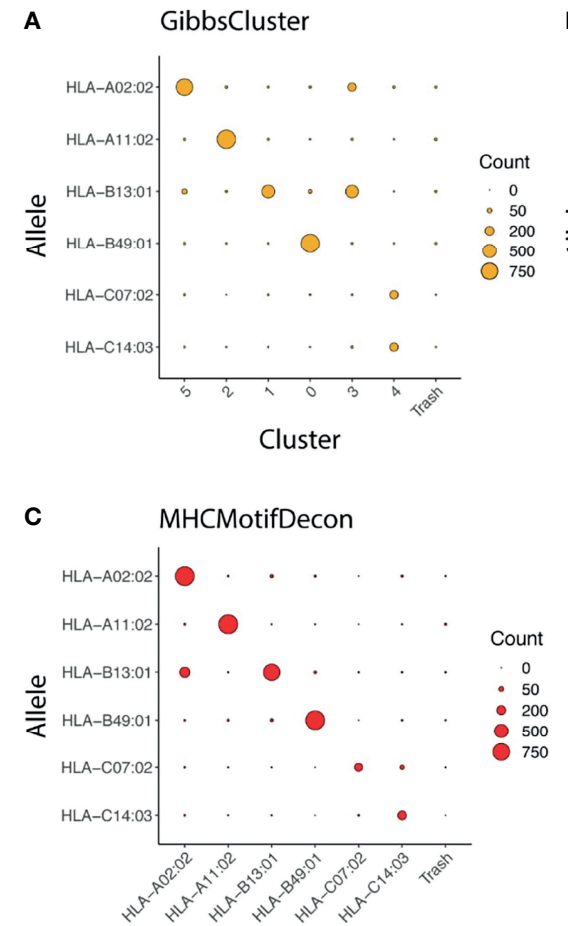

Allele
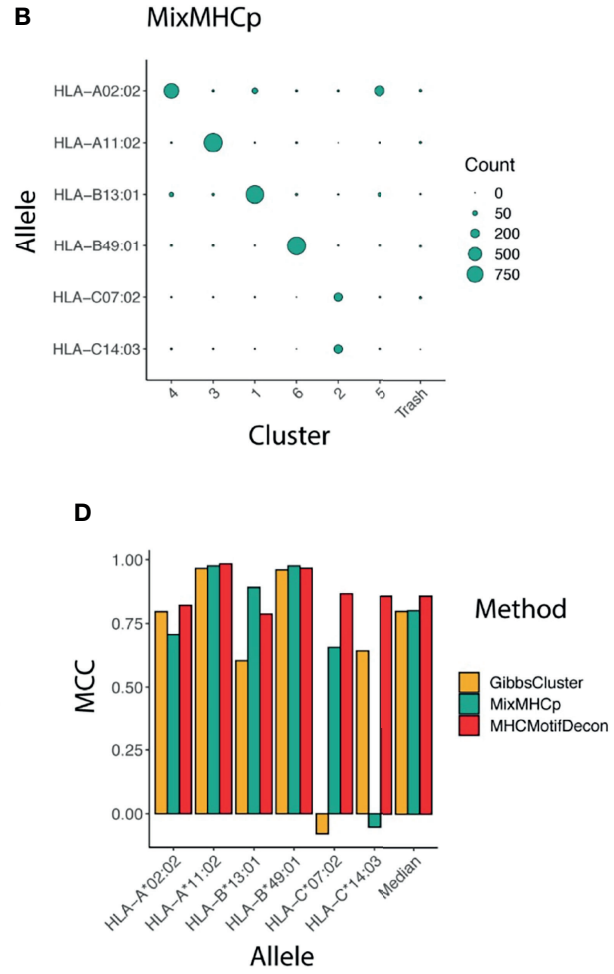

FIGURE 2 | Clustering confusion matrix and Matthews correlation performance for the three methods (A) GibbsCluster, (B) MixMHCp and (C) MHCMotifDecon. (D) Matthews correlation (MCC) performance estimates of accuracy of the motif deconvolution of the different methods for the artificial MS HLA eluted ligand dataset. MCC values for each method and HLA were estimated from the confusion matrices and cluster-HLA annotations shown in Figure 1.

FIGURE 3 | Motif deconvolution of the artificial dataset combining MS eluted ligand data from 4 cell lines each one expressing one individual HLA-DR allele. The three methods MHCMotifDecon, GibbsCluster (8), and Modec (10) were run as described in Materials and Methods. Eluted motif corresponds to GibbsCluster deconvolution of each of the single allele datasets using a single cluster ( $k=1)$.

again confirming the superior performance of MHCMotifDecon over the other two methods.

Similar to class I, a confusion matrix was constructed to quantify the deconvolution power of MHCMotifDecon compared to MoDec and GibbsCluster (Figure 4). This figure clearly confirms the superior performance of MHCMotifDecon over the two other methods with a close to 3 -fold increase in median performance compared to the second best method (MoDec). 


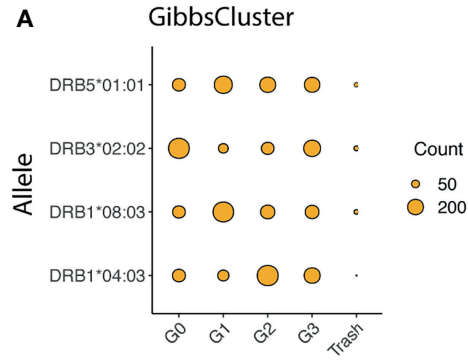

Cluster

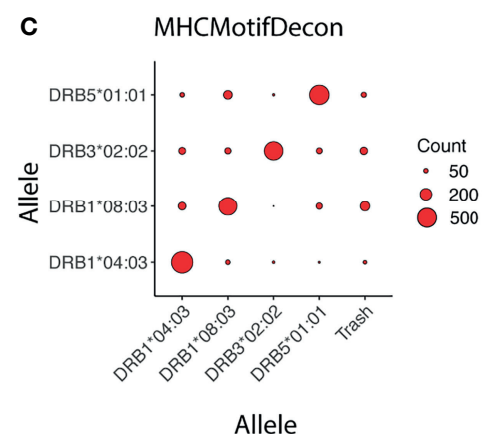

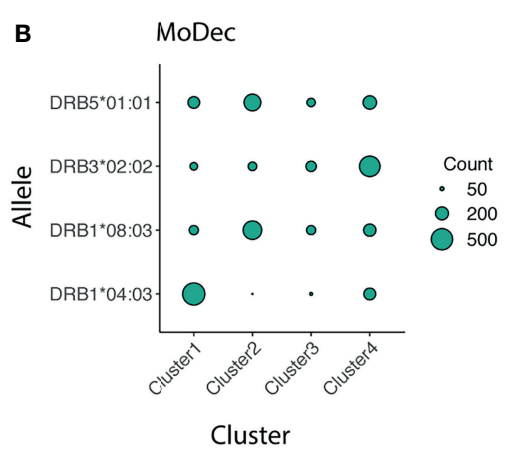

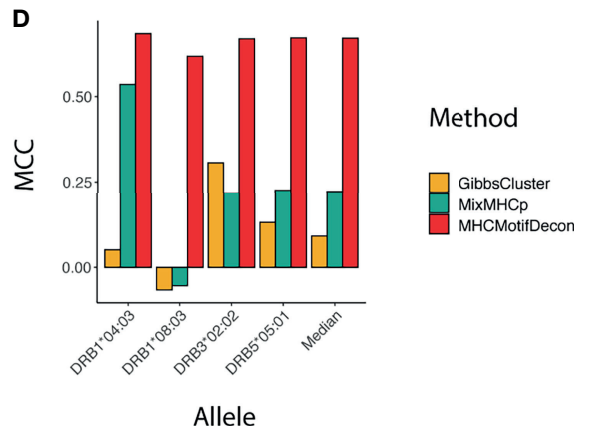

FIGURE 4 | Clustering confusion matrix and Matthews correlation performance for the three methods (A) GibbsCluster, (B) MoDec and (C) MHCMotifDecon. (D) Matthews correlation (MCC) performance estimates of accuracy of the motif deconvolution of the different methods for the artificial MS HLA eluted ligand dataset. MCC values for each method and HLA were estimated from the confusion matrices and cluster-HLA annotations shown in Figure $\mathbf{3}$.

\section{Dealing With Co-Immunoprecipitated MS Contaminants}

MS eluted ligand datasets generally contain a certain amount of co-immunoprecipitated contaminants. One example is the coimmunoprecipitation of varying amounts of HLA class I/II derived peptides that are non-specifically co-purified in addition to the HLA molecule of interest and contaminate the IP preparations (19). This problem, that happens despite the use of allele-specific antibodies, is inherent to the purification of membrane-bound HLA molecules and is due to the function of the detergent that disrupts the cell membrane and causes cell lysis. As a result, the purified HLA remains bound to a piece of membrane that carries other membrane proteins including other HLA alleles (19). This problem is inevitable unless cells that are engineered to secret the soluble form of the HLA are used (20, 21) which is not possible in case of tissues and biological samples. To illustrate the power of the tool to handle such contaminants, the complete immunopeptidome data obtained from the IHW09060 (CB6B) cell line expressing the HLA-DR alleles $\mathrm{DRB} 1^{\star} 13: 01$ and $\mathrm{DRB} 3^{*} 02: 02$ were submitted to MHCMotifDecon limiting the peptide length to 9-25 and excluding the use of the trash bin option (for details on the data generation refer to Materials and Methods). The result of this analysis (Figure 5A) showed an unusual motif and peptide length distribution for $\mathrm{DRB}^{\star}{ }^{\star} 13: 01$ with an additional peak taking up more than half of the peptide counts observed for the shortest peptide length; a clear sign of co-immunoprecipitated contaminants. Rerunning the analysis, setting the trash threshold to $20 \%$, the result in Figure 5B was obtained. In this figure, around $33 \%$ of the peptides were assigned to the Trash bin, and the motifs and length distribution for the two DR molecules now align with the expectations. To investigate the source of the large proportion of peptides assigned to the Trash bin, the complete IHW09060 peptide data was submitted to the MHCMotifDecon server, now selecting class $\mathrm{I}$, the class $\mathrm{I}$ alleles expressed in IHW09060 cell line (HLA-A*01:01, HLA-B ${ }^{\star}$ 15:01, HLA$\left.C^{\star} 03: 03\right)$, and setting a stringent threshold for the trash bin of $2 \%$. The result of the analysis, shown in Supplementary Figure 2, clearly, and in line with earlier works (22), indicates that the vast majority of the short peptides (in particular for length 8-11) have HLA class I origin. Next, the peptide data was resubmitted for class II motif deconvolution excluding peptides with predicted HLA class I restriction (Figure 5C). This analysis confirmed the clear motifs and expected peptide length distributions for the two DR molecules. Also, the analysis confirmed a very minor proportion of remaining MS contaminants (7\%), and a trash motif with a highly reduced information content. As a final analysis, the unfiltered data was submitted for class II motif deconvolution limiting the peptide length to 12-25 keeping all other parameters as default. The result of this analysis (Figure 5D) aligns closely with the result of the analysis excluding class I restricted peptides (Figure 5C) with the 


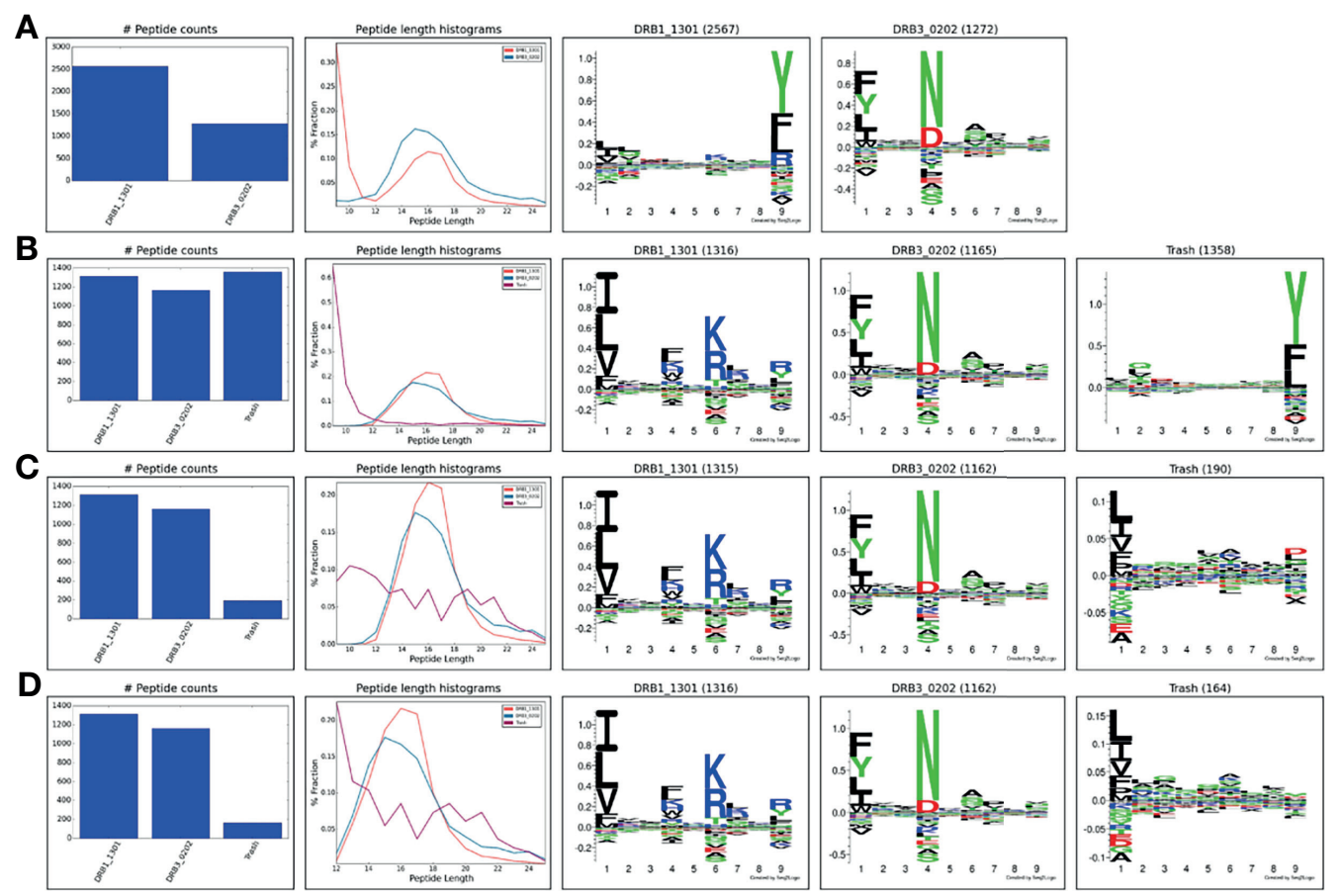

FIGURE 5 | HLA-DR motif deconvolution for the IHW09060 dataset using different strategies to deal with co-immunoprecipitated MS contaminants. (A) Peptide length 9-25, excluding the use of the trash bin option (achieved by setting the trash bin threshold to 101\%), (B) Peptide length 9-25, trash bin 20\%, (C) Peptide length 9-25, exclude class I binders, trash bin 20\%, (D) Peptide length 12-25, trash bin $20 \%$.

exception of the much larger amount of trash peptides found for peptides of length 12 in this latter analysis.

In summary, this analysis demonstrates the power of MHCMotifDecon to effectively perform HLA motif deconvolution also in immunopeptidome datasets with substantial amounts of co-purified contaminants. Furthermore, the analysis suggests that for class II motif deconvolution, placing a peptide length threshold of $12-25$ serves as an effective way to deal with class I co-immunoprecipitated contaminants. This however with the caution of potentially identifying a small proportion of false positive peptides at the shortest peptide length. To avoid this, the analysis suggests to filter out potential class I presented peptides prior to performing the class II motif deconvolution.

To further illustrate the issue of potential false positive coimmunoprecipitated MS contaminants, the motif deconvolution of the original data was compared to the deconvolution performed for a set of scrambled HLA-DR ligands (see Supplementary Figure 3 ). In the scrambled dataset, the peptide sequences have the same amino acid composition but in a shuffled order (for details refer to Methods section). In this comparison, the overlap between rank score in the original and scrambled datasets for HLA class II was found to be substantial for peptide lengths of less than 12 and above 21. In contrast, this overlap is basically absent for peptide lengths of 12-21 (Supplementary Figure 3B). Given this, for class II we recommend to filter the peptide length to fall in the range
12-21 when using MHCMotifDecon. Likewise for class I, we recommend applying a length filter to only include peptides of length 8-14 amino acids (see Supplementary Figure 3A).

\section{Characterization of the Peptide Repertoires of Primary and Secondary HLA-DR Molecules}

To fully demonstrate the application and power of the MHCMotifDecon method for analysis and interpretation of complex immunopeptidome datasets, a set of 11 HLA-DR immunoprecipitated immunopeptidome datasets were generated from homozygous B cell lines (see Supplementary Table 1). For details on the data generation refer to Materials and Methods. Next, this complete set of 11 HLA-DR immunopeptidome was analyzed using MHCMotifDecon with the purpose of characterizing the peptide repertoires of primary and secondary HLA-DR molecules in detail in the context of the contribution of the primary and secondary DR molecules to total DR peptidome, length distribution of each HLA-DR molecule, as well as the level of overlap between the DR molecules in each haplotype. Note, based on the conclusions from the above analyses, the data were first filtered using MHCMotifDecon to exclude peptides with predicted HLA class I restrictions. Then the peptide data were submitted to MHCMotifDecon together with information about their full HLA-DR typing using default MHC class II options and including length histograms and consistency matrix plots. The result of this analysis is shown in Figure 6. 
A
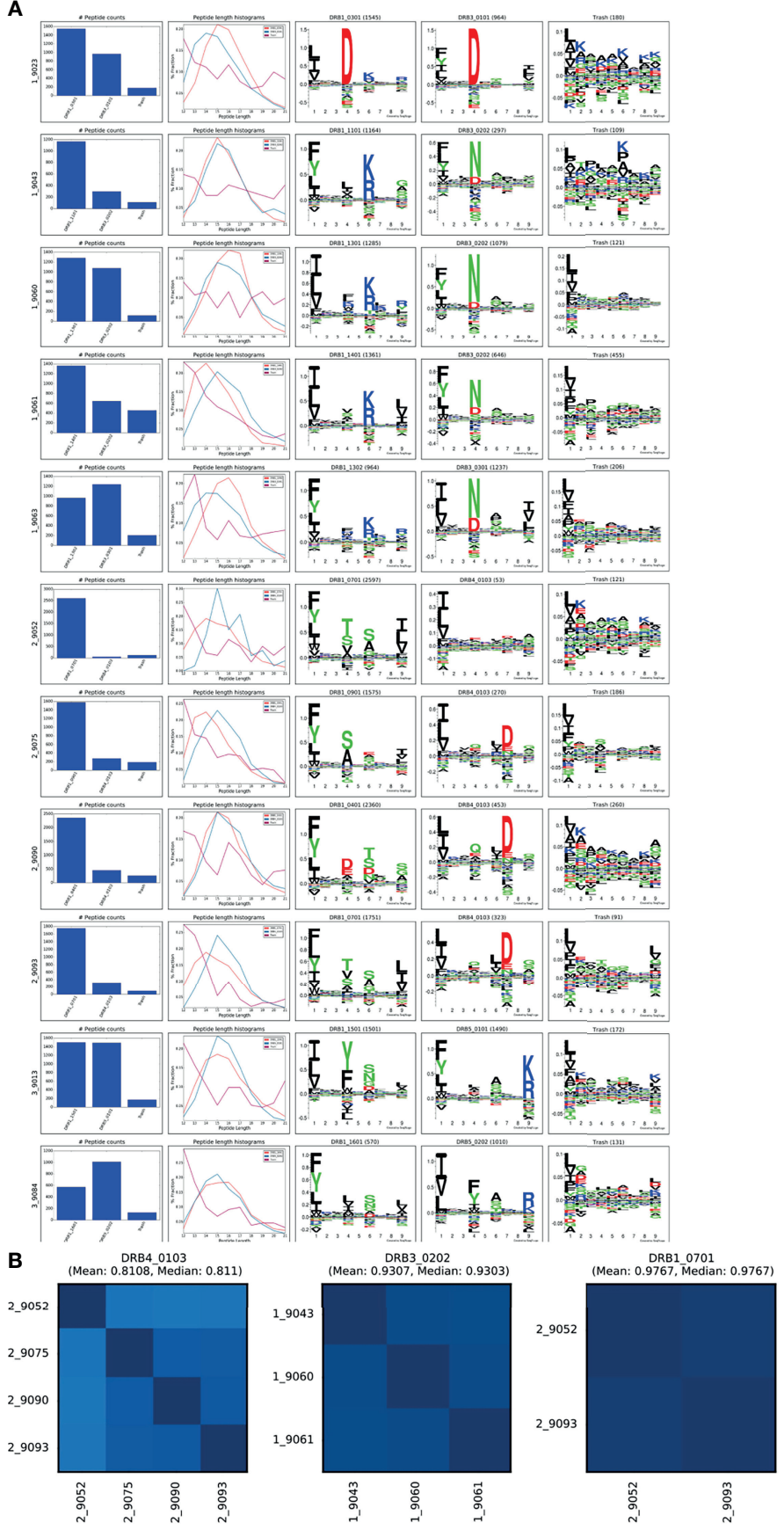

FIGURE 6 | MHCMotifDecon analysis for the MS peptidomics data obtained from the 11 cell lines. Peptide datasets were filtered for HLA class I binders as described in the text and submitted to MHCMotifDecon for motif deconvolution using default class II options. (A) Peptide counts, length distribution per alleles or trash cluster, and logos from HLA-DR alleles after deconvolution by the method. Each row corresponds to one dataset (cell line). The label $N_{-}$in front of each cell line (i.e., 1_9023) corresponds to the different haplotype groups (see Supplementary Table 1). The first column shows the number of peptides assigned to each HLA molecule and the Trash bin (containing peptides with a predicted rank $>20 \%$ ). The second column gives the peptide length distribution for each HLA, and Trash bin, and the remaining columns the binding motifs for each HLA molecule and Trash bin. Motifs are constructed from the predicted binding cores using Seq2Logo (23) with default settings. (B) Consistency matrices generated by the method for the three DR molecules shared between 2 or more cell lines using the method described in (15) defining the similarity between two HLA binding motifs in terms of the Pearson's correlation coefficient (PCC) between the two vectors of $9 * 20$ elements (9 positions and 20 amino acid propensity scores at each position). Note. that the consistency plot for DRB4*01:03 includes the null allele DRB4 ${ }^{\star} 01: 03 \mathrm{~N}$ expressed in the 9052 cell line. Removing this allele from the plot results in increasing the Mean PCC value to 0.88. 
Overall, these results demonstrated both the high quality of the generated MS HLA-DR immunopeptidome datasets (illustrated by the relative low proportion of peptides assigned to the trash cluster (Figure 6A), and a high consistency between the motif deconvolution for HLA-DR molecules shared between multiple cell lines (Figure 6B). Also, the figure illustrates the power of the MHCMotifDecon method to accurately deconvolute the DR motifs in these complex datasets (illustrated by the sharp motifs, and the well-defined peptide length distribution for the DR annotated peptides). As a side remark, the analysis further revealed subtle differences in length preference among various primary and secondary HLA-DR molecules. These differences will be discussed in more detail later.

\section{DRB5 Displays a Significant Contribution to the Total Immunopeptidome of the HLA-DR51 Haplotype Group}

DRB5 molecules are in linkage disequilibrium with DRB1 ${ }^{\star} 15$ and $\mathrm{DRB} 1^{\star} 16$ alleles in DR51 haplotype group. The B lymphoblastoid cell lines [SCHU (9013) and CALOGERO (9084)] express the $\mathrm{DRB}^{\star} 01: 01$ and $\mathrm{DRB}^{\star} 02: 02$ alleles in combination with $\mathrm{DRB} 1^{\star} 15$ and $\mathrm{DRB} 1^{\star} 16$ and therefore were selected to determine the contribution of DRB5 alleles to HLADR51 peptide repertoire. The motif deconvolution of the two cell lines shown in Figure 6 indicates the large contribution of both DRB5 molecules to the peptide repertoire of both cell lines. In fact, $\mathrm{DRB}^{\star} 01: 01$ and $\mathrm{DRB}^{\star}{ }^{\star} 02: 02$ contributed $50 \%$ (1490 out of a total of 2991 peptides) and $64 \%$ (1010 out of a total of 1580) to the total DR peptidome of 9013 and 9084 respectively (Supplementary Table 2).

Secondary DR alleles like primary DR molecules show a Bell shape and normal length distribution. When the length distribution of both DRB5 molecules were compared, both showed a preference for the 15 amino acid peptides.

The binding motif of $\mathrm{DRB} 1^{\star} 15: 01$ and $\mathrm{DRB} 5^{\star} 01: 01$ has been studied before by different groups using crystallography, binding assay and eluted peptides (24-28). We were able to refine the motif for the DRB5 alleles of HLA-DR51 haplotype, using a large set of peptides eluted from the homozygous BLCLs and identified by mass spectrometry. In all cases, the identified motif (see Figure 6) was in agreement with previously described motifs (25) with some additional residues in the anchor positions P1, P4, P6 and P9.

In DRB5 ${ }^{\star} 01: 01, \mathrm{P} 1$ showed preference for bulky aromatic and hydrophobic residues like Tyr (Y) and Trp (W) as well as Phe (F), Leu (L), Ile (I) and Val (V). At P4 it favors small amino acids like Leu (L), Ala (A), Ile (I) or Val (V). This secondary DR molecule accepts amino acids with aliphatic side chain like Ala (A), Ser (S), Gly (G), Pro (P) and Asn (N) at P6 and positively charged (basic) residues such as Lys $(\mathrm{K})$ or Arg $(\mathrm{R})$ at $\mathrm{P} 9$ that is essential for the interaction between the peptide and the $\mathrm{DRB}^{\star} 01: 01$ allele.

The same trend was observed in $\mathrm{DRB}^{\star} 02: 02$. This allele shared P6 and P9 amino acid preferences with DRB5 ${ }^{\star} 01: 01$. However, in DRB5 ${ }^{\star} 02: 02, \mathrm{P} 1$ has a strong preference for small amino acids like Ile (I), Val (V) and Leu (L), while P4 favors the bulky Tyr (Y), Trp (W) and Phe (F) amino acids. Therefore, $\mathrm{DRB}^{\star}$ 01:01 and 02:02 have different $\mathrm{P} 1$ and $\mathrm{P} 4$ preferences but share the same residues at P6 and P9.

While certain similar features can be identified between the motifs of the primary and secondary DR molecules in the DR51 haplotype, the difference between residue preference at $\mathrm{P} 1$ and P4, as well as a distinct basic P9 in both DRB5 molecules, has caused the peptide repertoire of the primary and secondary alleles in the DR51 haplotype to be complementary rather than overlapping (see Figure 7 and Supplementary Table 3).

\section{DRB4 Has a Limited Contribution to the DR53 Haplotype Group Peptide Repertoire}

DRB4 is associated with $\mathrm{DRB} 1^{\star} 04, \mathrm{DRB} 1^{\star} 07$ and $\mathrm{DRB} 1^{\star} 09$ in the DR53 haplotype group. Here, we investigated the peptide presentation by these 4 primary and secondary HLA DR using four different cell lines [AWELLS (9090), BER (9093), DKB (9075) and DBB (9052)]. The motif deconvolution results in Figure 6 indicate that DRB4 is presenting about 15\% of the total $\mathrm{DR}$ peptidome. That is, the primary DR molecules $\left(\mathrm{DRB} 1^{\star} 04\right.$, $\mathrm{DRB} 1^{\star} 07$ and $\left.\mathrm{DRB} 1^{\star} 09\right)$ contribute about 5-6 times more than
9013

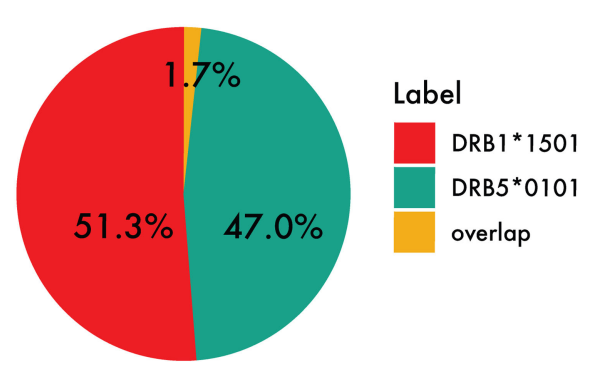

9084

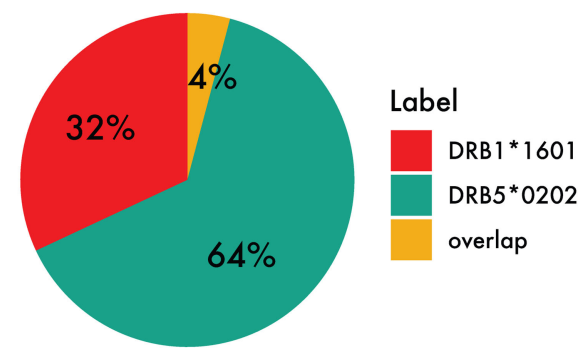

FIGURE 7 | Peptide overlap within the DR51 haplotype group. HLA-DR binding for MS ligands identified within the two DR51 cell lines (Left) 9013 (HLADRB1*15:01 and HLA-DRB5*01:01) and (Right) 9084 (HLA-DRB1*16:01 and HLA-DRB5*02:02) was predicted with NetMHCllpan-4.1 using a threshold of 1\% rank, and the relative contribution from each primary and secondary HLA-DR molecule as well as their overlap were calculated. 
DRB4 to the total peptide repertoire of DR53 haplotype (see Supplementary Table 2).

Despite the consistent contribution of DRB4*01:03 in 3 of the cell lines, we observed a below average presentation by this allele in the DBB (9052) cell line (2\%, 53 out of a total of 2597 DR annotated peptides). This proportion of presented peptides was comparable to the number of peptides assigned to the trash bin (121), and hence indistinguishable from the noise of the experiment. Using sequence-based typing method, we noticed a null phenotype for the $\mathrm{DRB} 44^{\star} 01: 03$ in this cell line. DRB4 ${ }^{\star} 01: 03 \mathrm{~N}$ is characterized by a splice site mutation at the $3^{\prime}$ end of the first intron (AG > AA), leading to a transcript that is larger than that of the common DRB4 molecule and believed to be processed into a non-functional HLA molecule (29). Our data, which is in agreement with the typing results, suggest that this null allele is non-functional or have retained a very limited antigen presentation capacity.

In primary DR molecules, both $\mathrm{DRB} 1^{\star} 07: 01$ and $\mathrm{DRB} 1^{\star} 09: 01$ showed a preference for shorter peptides (14 mers), while $\mathrm{DRB}^{\star}{ }^{*}$ 4:01 and DRB4 ${ }^{\star}$ 01:03 showed the common class II length preference for 15 amino acid peptides.

The motif for the DRB4*01:03 is distinct as it displays 5 key anchor positions (P1, P4, P6, P7 and P9) instead of the conventional 4 anchor positions (P1, P4, P6 and P9). P1 has preference for Leu (L), Ile (I), Val (V), followed by Phe (F) and Met (M). P4 favors Gln (Q) and Glu (E), and less Val (V), Ala (A) and Leu (L). P6 accepts amino acids with hydrophobic side chains like Leu (L), Val (V) and Ile (I). P7 which is unique to DRB4 has strong preference for negatively charged or uncharged polar side chains including Asp (D), Glu (E) and Asn (N) and finally P9 that shows preference for Gly $(G)$, Ser (S) and Ala (A).

When we compared the motif of the DRB $4^{\star} 01: 03$ with the associated primary DR alleles (DRB1 ${ }^{\star} 04: 01, \mathrm{DRB} 1^{\star} 07: 01$ and $\left.\mathrm{DRB} 1^{\star} 09: 01\right)$, we noticed that in all combinations the primary DR alleles have a $\mathrm{P} 1$ position that favors the large aromatic residues like Phe (F), Tyr (Y) and Trp (W) (Figure 6). DRB4restricted epitopes, on the other hand, tend to contain smaller amino acids (Leu, Ile, Val) at the P1 anchor position.

This lack of similarity in the amino acid preference at P1, which is considered a major determinant of peptide selection, in addition to the $5^{\text {th }}$ anchor position (P7) with strong preference for acidic residues, which is absent in all other DR alleles, explains the difference between the peptide repertoires of primary and secondary DR alleles in DR53 haplotype. In fact, except for $\mathrm{DRB} 1^{\star} 04: 01$ and $\mathrm{DRB} 4^{\star} 01: 03$ that share some similarity and favor negatively charged residues at $\mathrm{P} 4$, the other members of the DRB53 haplotype have little to no similarity in their motifs which results in an extremely low overlap between the ligandome of the $\mathrm{DRB} 1^{\star} 07$ and $\mathrm{DRB} 1^{\star} 09$ with the DRB4*01:03 (see Supplementary Table 3).

\section{DRB3 Contribution to Total DR52 Peptide Repertoire Varies Considerably}

The DRB3 gene encodes 3 major alleles: DRB3 ${ }^{\star} 01: 01$, $\mathrm{DRB}^{\star} 02: 02$, and $\mathrm{DRB}^{\star}{ }^{\star} 03: 01$ that display a strong association with $\mathrm{DRB} 1^{\star} 03, \mathrm{DRB} 1^{\star} 11, \mathrm{DRB} 1^{\star} 12, \mathrm{DRB} 1^{\star} 13$ and $\mathrm{DRB} 1^{\star} 14$ in the DR52 haplotype family. The cell lines [VAVY (9023), BM21
(9043), CB6B (9060), 31227 ABO (9061) and WT47 (9063)] express frequent variants of DRB3 alleles including $\mathrm{DRB} 3{ }^{\star} 01: 01$, $\mathrm{DRB}^{\star} 02: 02$ and $\mathrm{DRB} 3{ }^{\star} 03: 01$ in combination with $\mathrm{DRB} 1^{\star} 03: 01$, $\mathrm{DRB}^{\star} 11: 01, \mathrm{DRB} 1^{\star} 13: 01, \mathrm{DRB} 1^{\star} 13: 02$ and $\mathrm{DRB} 1^{\star} 14: 01$. We examined the peptide presentation and contribution of different DRB3 molecules to the total DR52 peptide repertoire using above cell lines. We observed a substantial variation in the contribution of the DRB3 molecules to the total DR immunopeptidome ranging from 20 to 56 percent (Supplementary Table 2). This variation was not only seen among different DRB3 variants but also in one variant (DRB3 $\left.{ }^{\star} 02: 02\right)$ in combination with different primary DR alleles $\left(\mathrm{DRB1}^{\star} 11: 01,13: 01 \& 14: 01\right)$. Whether this broad range of contribution is an inherent characteristic of this DRB3 allele or the accompanying primary DR molecules play a role in this, remains to be further investigated.

The overall contribution of DRB3 is more than what was observed for DRB4 (Mean: 15.4\%). In some haplotypes, where $\mathrm{DRB} 3$ is co-expressed with $\mathrm{DRB} 1^{\star} 13: 01$ and $\mathrm{DRB} 1^{\star} 13: 02$ alleles, the two DRB3 molecules (DRB3 ${ }^{\star} 02: 02$ and $\mathrm{DRB}^{\star} 03: 01$ ) comprise between $46 \%$ and $56 \%$ of the total DR peptide repertoire respectively, similar to what we observed with DRB5 variants (Mean: 56.8\%) (Supplementary Table 2).

When comparing the length distribution of the 3 DRB3 molecules (Figure 6), we found an interesting pattern in which the $\mathrm{DRB}^{\star}{ }^{\star}$ 02:02 showed the normal class II preference for 15 mers whereas a shift toward shorter peptides with a preference for 14 mers was observed for $\mathrm{DRB}^{\star} 01: 01$ and $\mathrm{DRB} 3^{\star} 03: 01$.

In primary $\mathrm{DR}$ molecules, we observed a wider range of deviation from the general class II length preference. $\mathrm{DRB} 1^{\star} 13: 01$ and $\mathrm{DRB} 1^{\star} 13: 02$ showed a preference for longer peptides (16 mers), while $\mathrm{DRB} 1^{\star} 03: 01$ and $\mathrm{DRB} 1^{\star} 11: 01$ showed the common class II length preference for 15 mers, and $\mathrm{DRB}^{\star}{ }^{\star} 14: 01$ favored shorter peptides (14 mers).

Next, we investigated in detail the binding motifs obtained for the three DRB3 molecules included in our dataset. DRB3 ${ }^{\star} 01: 01$ motif consists of only 3 anchor positions (30). P1 in DRB3 ${ }^{\star} 01: 01$ has a preference for large, aromatic amino acids like Tyr (Y) and Trp (W) as well as Phe (F), Leu (L) and Ile (I). At P4 position, $\mathrm{DRB}^{\star}$ 01:01 shows a distinct preference for Asp (D), which is a negatively charged amino acid. $\mathrm{DRB} 1^{\star} 03: 01$, which is in linkage disequilibrium with $\mathrm{DRB}^{\star}{ }^{\star} 01: 01$ shares the very same preference at $\mathrm{P} 4$. In crystallography experiments, pocket $\mathrm{P} 4$ has shown a strong positively charged character and therefore the preference for aspartic acid at $\mathrm{P} 4$ is consistent with suggested positive charge of the pocket (30).

Due to the extremely small and shallow P6 pocket in the crystal structure, P6 in DRB3 ${ }^{\star} 01: 01$ can only accommodate small residues which has resulted in a "P1-P4-P9" binding motif (30). P9 in DRB3 ${ }^{\star} 01: 01$, does not show a specific preference and can accept small amino acids like Leu (L), Ile (I), Phe (F) and Val (V).

The DRB3 ${ }^{\star}$ 03:01 molecule shares a series of conserved elements with other DR alleles but has some unique features in the peptide binding groove. In P1, it prefers the amino acids with a small aliphatic side chain like Leu (L), Ile (I) and Val (V). This is different from the $\mathrm{P} 1$ in the $\mathrm{DRB} 3^{\star} 01: 01$, that can accept large aromatic residues like $\operatorname{Tyr}(\mathrm{Y})$ and $\operatorname{Trp}(\mathrm{W})$ in addition to Phe 
(F), Leu (L) and Ile (I). At P4, DRB3*03:01 has a strong preference for asparagine (N) and aspartic acid (D) which fits in the hydrophilic pocket in the crystal structure of the molecule. In contrast to P4, P6 does not seem to have preference for any specific amino acid and can accept residues with small side chains like Pro (P), Ala (A), Val (V) and Gly (G). P9 in both $\mathrm{DRB}^{\star}{ }^{*} 01: 01$ and $\mathrm{DRB}^{\star}{ }^{\star} 03: 01$ alleles are very similar and favor small amino acids like Leu (L), Ile (I) and Val (V). P1 and P4 appear to be the key determinants of specific binding in DRB3 $^{\star}$ 03:01 allele (31).

The crystal structure of the $\mathrm{DRB}^{\star} 02: 02$ is not available (31). Comparing the motif of this DRB3 variant with the DRB3 ${ }^{\star} 01: 01$ and $\mathrm{DRB}^{*}{ }^{*}$ 3:01 shows that this molecule shares an identical P4 with the $\mathrm{DRB}^{*} 03: 01$ and displays the same amino acid preference for $\mathrm{P} 1$ as $\mathrm{DRB}^{\star} 01: 01$. It also shares a similar amino acid composition at P6 with DRB3 ${ }^{\star} 03: 01$. However, the P9 in this molecule is different from both DRB3 ${ }^{\star} 03: 01$ and $\mathrm{DRB}^{\star}{ }^{\star} 01: 01$ as it favors smaller residues.

Comparison of these three major HLA-DRB3 alleles suggests that they were derived from one another by recombination events that rearranged the four major peptide-binding pockets at peptide positions $1,4,6$, and 9 (31).
When the motif of these DRB3 molecules was compared with their associated primary DR alleles, except for $\mathrm{DRB} 1^{\star} 03: 01$ that shares the same preference at $\mathrm{P} 4$ with the $\mathrm{DRB} 3{ }^{*} 01: 01$, the rest of the primary DR molecules showed different preferences in P1 and $\mathrm{P} 4$ and all presented positively charged residues Lys $(\mathrm{K})$ and Arg (R) at P6. Therefore, except for the large overlap that is observed between the peptide repertoire of the $\mathrm{DRB} 1^{\star} 03: 01$ and $\mathrm{DRB}^{\star}{ }^{*} 01: 01$, in the rest of the haplotypes the difference between $\mathrm{P} 1$ and P4 preferences has caused the peptidome of the DRB3 and the accompanying DRB1 alleles to diverge and the overlap to be minimal (see Supplementary Table 3).

\section{Deconvolution of DR Ligandome From Complex Biological Samples}

Finally, to evaluate the performance of the MHCMotifDecon method on deconvolution of the HLA-DR peptides identified directly from scarce biological samples with complex, heterozygous DR type, clinical specimens from patients with cancer (Chronic lymphocytic leukemia and Lymphoma) and autoimmune diseases (Rheumatoid Arthritis, Lyme Arthritis and Sarcoidosis) obtained from previously published datasets were analyzed (Figure 8) (32-35).
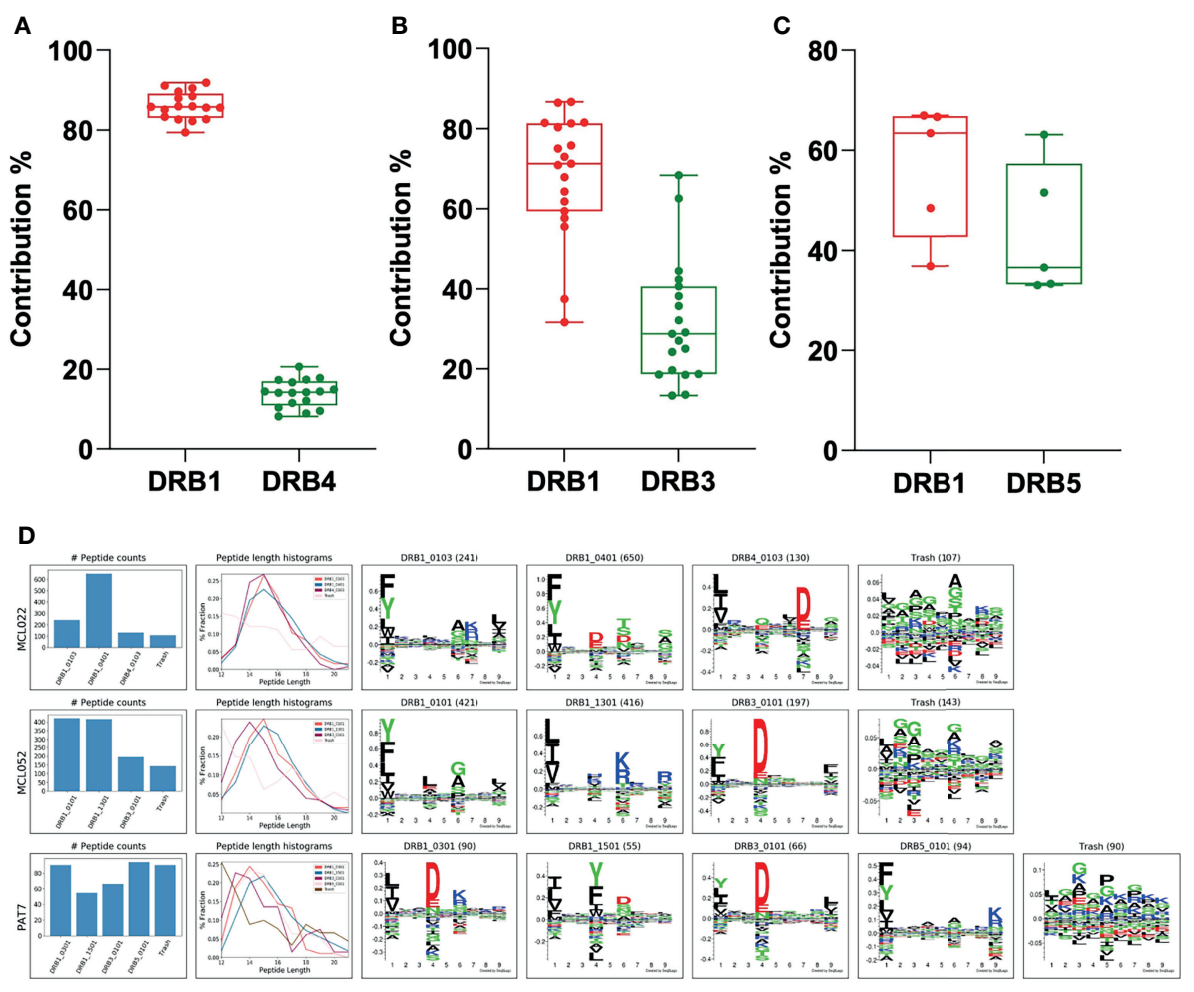

FIGURE 8 | Contribution of the DRB3, 4 and 5 alleles vs. DRB1 in biological samples. (A) Contribution of DRB4 vs. DRB1 was calculated using biological samples from ten patients with mantle cell lymphoma (MCL), one patient with chronic lymphocytic leukemia (CLL), three patients with rheumatoid arthritis (RA) and two patients with Lyme disease (LA). (B) Contribution of DRB3 vs. DRB1 was calculated using samples from nine patients with mantle cell lymphoma (MCL), two patients with chronic lymphocytic leukemia (CLL), one patient with rheumatoid arthritis (RA), four patients with Lyme disease (LA) and one patient with Sarcoidosis. (C) Contribution of DRB5 vs. DRB1 was calculated using samples from two patients with rheumatoid arthritis (RA), two patients with Lyme disease (LA) and one patient with Sarcoidosis. In all cases, the DRB3, 4 or 5 with the highest LD was assigned to the DRB1 before deconvolution of the DR peptidome by the MHCMotifDecon. (D) Peptide counts, length distribution per alleles or trash cluster, and logos from deconvolution of HLA-DR peptidome of the patients after assigning DRB3, 4 and 5 to the associated DRB1 alleles. Each row represents one patient. 
Overall, while the clinical samples investigated here included a wide variety of tissues and cell types such as lymph node biopsies, splenectomy, synovial tissues and fluid, PBMC and bronchoalveolar lavage cells with different antigen presentation capacities, we observed that the contribution of the DRB3, 4 and 5 molecules in peptide presentation aligns extremely well with our findings from homozygous B cell lines, with DRB4 showing the lowest contribution with the least variation (Figure 8A), DRB3 presenting a large variation in agreement with our observations from the cell lines (Figure 8B) and lastly, DRB5 that displays the highest contribution to the accompanying DRB1 allele (Figure 8C). It is worth mentioning that the data presented here is the contribution of DRB3, 4 and 5 to the accompanying DRB1 only and not to the total DR immunopeptidome in each patient.

\section{DISCUSSION}

Analyzing and interpreting complex immunopeptidome data is a highly challenging and intricate task considering the expression of multiple HLA alleles on the cell surface and the presence of coimmunoprecipitated contaminants. Here, we have proposed a simple yet very powerful tool, called MHCMotifDecon, to assist the computationally inexpert users in performing this task. The tool takes the peptide sequences from MS immunopeptidomics experiment(s) together with the associated full HLA typing from one or more samples as input and performs a complete motif deconvolution of the data assigning each peptide to its most likely HLA restriction element while removing probable MS coimmunoprecipitated contaminants.

Using artificial datasets constructed from peptides with known HLA restriction, the method was demonstrated to outperform other available motif deconvolution tools in particular for low expression alleles such as HLA-C in case of HLA class I, and in general across all alleles for HLA class II.

In order to illustrate the application of this tool, the contribution of the primary and secondary DR molecules to total DR peptidome as well as length distribution of each HLADR molecule was investigated in detail in a large set of immunopeptidome data generated for this study from a panel of homozygous B lymphoblastoid cell lines (BLCL) selected with an HLA-DR profile that covers the most frequent variants of the secondary DR alleles in the haplotypes with high population coverage. We next looked into the contribution of these secondary DR alleles in clinical samples from autoimmune and cancer patients with heterozygous DR HLA type. Our results from these analyses, which are in mutual agreement, show that the secondary DR molecules contribute to the class II DR immunopeptidome far more than what was previously assumed. The ligands presented by secondary DR alleles not only expand the DRB1 peptide repertoire, they might also add an independent function to the haplotype they contribute to (36). Among the secondary DR molecules studied here, DRB5 demonstrated the highest contribution to the class II ligandome. Next was DRB3, followed by DRB4 that displayed the lowest level of peptide presentation. Interestingly, we found that in some haplotypes (DR51 and DR52) DRB5 and DRB3 contribute even more than the accompanying DRB1 molecule. DRB4 was the only molecule that consistently contributed less than the DRB1 alleles in the DR53 haplotype family. These findings are in agreement with the previous studies performed on a limited number of haplotypes at transcriptional and mRNA expression level, where, for DRB3 (only DRB3*01:01 and $\mathrm{DRB}^{\star}{ }^{*}$ 02:02 included) the relative proportion of mRNA was reported to vary from being four times lower to almost equal to the amount of accompanying DRB1 mRNA (37). DRB5 mRNA was found to be more abundant than the $\mathrm{DRB} 1^{\star} 15$ (38), whereas the level of DRB4 mRNA was as much as seven times lower than DRB1*07 $(39,40)$.

While we observed a considerable degree of variation in the contribution of DRB3 molecules to the DR52 peptidome, these molecules contributed the most when expressed in combination with the $\mathrm{DRB} 1^{\star} 13: 01$ and 13:02 primary DRs. HLA-DRB1 ${ }^{\star} 13$ alleles have been shown to play a protective role against multiple autoimmune and certain infectious diseases like HBV in HLADisease association studies (41-43). It has been hypothesized that the protective role of these DR molecules is due to more efficient antigen presentation and promoting deletion of autoreactive $\mathrm{T}$ cells during thymic selection (41). However, our results show that the accompanying DRB3 molecule present a significant proportion of the peptidome when co-expressed with $\mathrm{DRB}^{\star}{ }^{\star} 13$ molecules suggesting that DRB3 molecules that generously add to breadth and diversity of the peptide repertoire, may play a part in the universal protective nature of the $\mathrm{DRB} 1^{\star} 13$ alleles.

On the other end of the spectrum there are DRB1 alleles that are associated with DRB4 molecules. One such allele is $\mathrm{DRB} 1^{\star} 04: 01$ that is a risk factor for type 1 diabetes (T1D) and rheumatoid arthritis (RA) (44). Our results, which was confirmed by deconvoluting the DR ligandome of the clinical samples from RA patients, indicate that DRB4 molecules, due to their limited repertoire, do not contribute to the diversity and extent of the haplotype total peptidome at the same level as DRB3 and DRB5.

Another important finding of this study is that the Secondary DR peptide repertoire complements the peptidome of the DRB1 molecules and is not redundant. The complementarity or redundancy of the peptide repertoires of the DRB3, 4 and 5 alleles has long been a source of controversy and overlap between the peptide repertoires of the $2 \mathrm{DR}$ molecules in a given haplotype has been reported by different groups $(45,46)$. However, our results demonstrate that the overlap between the DR molecules in all the haplotypes studied here is minimal with the only exception being the $\mathrm{DRB} 1^{\star} 03: 01$ and $\mathrm{DRB} 3^{\star} 01: 01$. These findings are supported by differences in the motifs and amino acid preferences in different anchor positions. Some of these differences can be seen consistently in all haplotypes and are not allele specific like $\mathrm{P} 1$ and $\mathrm{P} 4$ pockets that exhibit distinct binding preferences.

The P1 pocket in the crystal structure of the HLA-DR molecules is dimorphic and contains either glycine or valine at position 86 of the B chain. Presence of glycine produces a larger pocket which results in a preference for bulky hydrophobic and aromatic residues. Substitution of glycine with valine creates a 
shallow hydrophobic pocket that is only able to accommodate small hydrophobic amino acids $(47,48)$. Intriguingly we found that in all haplotypes examined here, except for (DRB1*11:01, $\mathrm{DRB}^{\star} 02: 02$ ), where one DR allele (either primary or secondary) has a large $\mathrm{P} 1$ pocket and prefers the bulky aromatic residues like Tyr (Y) and Trp (W), the second DR allele favors the small aliphatic residues like Ile (I), Lue (L) and Val (V). This is in agreement with $\mathrm{P} 1$ pocket role in determining allele specific preferences for anchor residues (47) and explains how this structural and steric hindrance at $\mathrm{P} 1$ pocket causes the primary and secondary DR alleles to present diverse peptidome with minimal overlap.

The $\mathrm{P} 4$ pocket is the most diverse and polymorphic pocket of the HLA-DR binding site (27) which results in the different residue preferences at $\mathrm{P} 4$ in the peptide repertoire of $\mathrm{DR}$ molecules in a haplotype. This is another factor that contributes to the presentation of distinct peptidomes by primary and secondary DR molecules. The significance and impact of the position 4 in creating a diverse peptidome is even more highlighted when we appreciate the large degree of overlap between $\mathrm{DRB} 3{ }^{\star} 01: 01, \mathrm{DRB} 1^{\star} 03: 01$ which is mainly due to sharing the identical P4 anchor residue Asp (D) for the two molecules. It has been suggested that the P4 pocket of HLA-DR makes an important contribution to susceptibility to different human autoimmune diseases (27).

In addition to the distinct residue preferences at P1 and P4 that cause the peptidome of the primary and secondary DR alleles to be different from each other in all haplotypes, there are other distinguishing features that are unique to certain haplotypes. For example, in the DR53 haplotype, the additional anchor position (P7) in DRB4*01:03, imposes very different peptide binding requirements between the primary and secondary DR molecules explaining why DRB4 displays the least number of overlapping peptides with the accompanying DR7 and DR9 molecules.

In the end, the non-redundant and complementary nature of the secondary DR peptidomes is not unexpected as throughout the evolution different types of HLA have been developed by recombination events, to add diversity to the immune system and better defend the body against external pathogens. Our data indicate that the secondary DR peptide repertoire in all haplotypes expands the peptidome of the DRB1 molecules $(26,49)$. More importantly these peptides similar to their accompanying DRB1 presented peptides can activate autoreactive $\mathrm{CD} 4^{+} \mathrm{T}$ cells and provoke an autoimmune response which has been demonstrated by multiple studies where the DRB3, 4 and 5 restricted T cells are detected in measurable amount in patients with infectious and autoimmune diseases $(49,50)$.

In summary, given the extent of contribution of the secondary DR alleles, their complementary and non-redundant nature, in addition to their ability to successfully provoke a $\mathrm{T}$ cell response reveal the significance of these molecules and strongly suggest that they should be regarded as functionally independent alleles in future studies of autoimmune and infectious diseases.

The proposed MHCMotifDecon tool is supervised and performs the motif deconvolution using predicted HLA restrictions. While this, as demonstrated here, provides a highly powerful and successful approach for motif deconvolution and handling of complex MS peptidomics datasets, one should bear in mind that these successes are contingent on accurate binding prediction power being available for the MHC molecules in question. Also, one should note that the tool makes unambiguous assignment of HLA restrictions, hence failing to report multi-allele binding of promiscuous peptides.

With these cautions in mind, in conclusion, we have proposed a novel and powerful tool allowing computationally novice users to analyze and interpret complex immunopeptidome datasets, enabling accuracy assessment, motif deconvolution and HLA restriction assignment, estimation of relative allele contribution, and length profile investigations. The tool was demonstrated to achieve state-of-the-art performance and illustrated to enable novel biological discoveries. Given its ease of use, we expect the tool to be highly valuable for the scientific community facilitating better use and understanding of immunopeptidome data.

\section{MATERIALS AND METHODS}

\section{MHCMotifDecon Method}

Input peptides are first filtered by length range (determined by the user) with default lengths (8-14) for MHC class I and (1321) for MHC class II. All the remaining unique peptides are then predicted for MHC presentation towards all the MHC alleles expressed in the given cell line using NetMHCpan4.1 (16) or NetMHCIIpan (a retrained version for this paper). NetMHCpan and NetMHCIIpan methods can make a prediction for all MHC molecules with known protein sequence. A key prediction value from the two methods is the percentile rank score for the likelihood of a peptide being presented by a given MHC molecule. This score reflects the likelihood of a random natural peptide obtaining a prediction score equal to or higher than the score for the given peptide. This score hence ranges from 0 $100 \%$, where 0 corresponds to the strongest possible score exceeding the score for all random natural peptides. For MHC class I, a percentile rank value of $2 \%$ or lower is considered a binder, and for class II the corresponding value is 5\% (16). Likewise, earlier work has shown that peptides with a percentile rank score $>20 \%$ are MS co-immunoprecipitated contaminants (25). Given this, by default all the peptides with a higher rank score of $20 \%$ to all the proposed alleles are considered as a nonbinder or contaminant, and therefore assigned to the trash cluster. Optionally, threshold can also be tuned by the user to be more or less stringent.

The graphical output of the tool provides: sequence motif logos for all the present alleles with a minimum of 10 (default value) peptides assigned to it, the length distribution for all the alleles and the trash cluster, and a bar-plot with peptide counts assigned to each of the clusters. In addition, a correlation matrix can be plotted when analyzing several samples with the same HLA alleles on the same run, to check for consistency. The correlation matrices are then calculated by stacking all the amino 
acid frequencies for all the core positions in a vector of $20^{\star} 9$ and obtaining a Pearson correlation coefficient (PCC) among the method information and the sample data. Additional text datafiles provided in the output can be downloaded after the analysis and include the raw and rank scores for each peptideallele combination, and the 9-mer core peptide sequence. Further details about the use of the tool is available at the instruction tab at the web-server.

\section{Retrain of NetMHCIlpan}

NetMHCIIpan was specifically retrained for this publication (updated version called NetMHCIIpan-4.1) excluding all single allele data from $\mathrm{DRB} 1^{\star} 04: 03, \mathrm{DRB} 1^{\star} 08: 03, \mathrm{DRB}{ }^{\star} 02: 02$ and $\mathrm{DRB}^{\star}$ 01:02 alleles. Single allele data for those alleles were left out to be used for the independent benchmark of MHCMotifDecon. Compared to NetMHCIIpan-4.0, additional single allele datasets from Abelin et al. publication were included (7), and the binding affinity data were re-curated to include updated data for DQ4. The data used to retrain this method is available at https://services. healthtech.dtu.dk/suppl/immunology/NetMHCIIpan-4.1. Apart from the modified data, the method is identical to the previously published version of NetMHCIIpan 4.0 (16). A performance comparison of NetMHCIIpan-4.1 and 4.0 is included in Supplementary Figure 1 demonstrating a significantly improved performance of version 4.1. NetMHCIIpan-4.1 is available at https://services.healthtech.dtu.dk/service.php?NetMHCIIpan-4.1.

\section{Benchmark Datasets}

Artificial MS MHC class I eluted ligand dataset. Peptides of length 8-14 amino acids were collected from the Abelin et al. publication (5). Peptides sharing an 8 mer or longer overlap with the NetMHCpan-4.1 training data were excluded. A random subset of 1000 peptides were sampled from the HLA-A ${ }^{\star} 02: 02$, HLA-A $^{\star}$ 11:02, HLA-B ${ }^{\star}$ 13:02 and HLA-B ${ }^{\star}$ 49:01 single allele datasets, and 200 peptides were sampled from HLA-C ${ }^{\star} 07: 02$, and $\mathrm{HLA}-\mathrm{C}^{\star} 14: 03$ datasets.

Artificial MS MHC class II eluted ligand dataset. Peptides with single allele associations for MHC class II were retrieved from MassIVE, MSV000083991 (7). Eluted ligands from cell lines expressing only one of the alleles $\mathrm{DRB} 1^{\star} 04: 03, \mathrm{DRB} 1^{\star} 08: 03$, $\mathrm{DRB}^{\star}{ }^{\star 02: 02}$ and $\mathrm{DRB}^{\star} 01: 02$ were used to generate the artificial dataset for class II. The four single allele datasets were excluded from NetMHCIIpan retraining performed for this paper, to avoid overlapping peptides present on the test and the train set. Additionally, peptides that shared a stretch of 9 amino acids with the dataset used for training NetMHCIIpan were excluded. Finally, for each of the alleles mentioned before, 800 unique peptides of length 13-21 were combined to generate the artificial dataset for MHC-II.

\section{Deconvolution of Immunopeptidome Data}

MHCMotifDecon was used with the default parameters for class I artificial dataset (Length range $=8-14$; Class $=\mathrm{I}$; Threshold for trash cluster $=20$ ) and for class II artificial dataset (Length range $=$ 13-21; Class = II; Threshold for trash cluster $=20$ ). For the inhouse MHC class II datasets, the length range was expanded to 1221 to further analyze length specificities.
GibbsCluster deconvolution for MHC class I artificial dataset was performed using default parameters as described earlier (8), and including 1-6 clusters, motif length 9 amino acids, using "trash cluster" with a threshold=2, performing single sequence moves at every iteration, max length deletions $=5$, max length insertions $=1$, number of seeds for initial starting conditions $=5$, Number of iterations $\mathrm{x}$ sequence $\mathrm{x}$ temperature step $=50$.

The deconvolution softwares MixMHCp and MoDec were downloaded from the GitHub repositories (https://github.com/ GfellerLab/MixMHCp and https:/github.com/GfellerLab/ $\mathrm{MoDec})$ and run with default parameters for class I and class II $(10,18)$.

As MHCMotifDecon by construction will identify clusters equal to the number of HLA alleles defined in the sample, the number of clusters for the deconvolution of the artificial immunopeptidome datasets was set to six for MHC class I and four for MHC class II for MixMHCp and GibbsCluster methods included in the comparison. Also, as MixMHCp and GibbsCluster only perform motif clustering and do not assign HLA allele to the identified clusters, the HLA assignment for these methods was performed by visual inspection in unambiguous cases and by performance optimization in ambiguous cases.

\section{Scrambled Dataset Generation}

All MS peptide data from eleven cell lines in the in-house MHC class II datasets were merged $(\mathrm{N}=49,550)$. Each peptide sequence was scrambled 100 times and predicted with NetMHCIIpan-4.1 for all the alleles expressed by the cell line presenting the original peptide sequence. For each peptide-scramble combination the best (lowest) rank score was selected. A random sample of the scrambled peptide data was used to match the counts of the original dataset following the same length distribution.

\section{Performance Metrics and Statistical Significance}

A Matthews coefficient correlation (MCC) was calculated for each prediction method and allele, from the confusion matrix constructed from the complete motif deconvolution matrix (like the ones shown in Figure 3) assigning the true positive count (TP) as the number of peptide sequences from the Allele_X dataset assigned to Cluster_X, the false positives count (FP) to the number of peptides assigned to Cluster_X but belonging to any of the remaining alleles in the sample and excluding the trash cluster. Finally, the false negative (FN) count was assigned as the number of peptides from the Allele_X dataset assigned to any of the remaining Clusters, and the true negative (TN) count as the number of peptides that were correctly predicted as non-binders to Allele_X. For GibbsCluster, MixMHCp and MoDec that do not provide an allele association, the cluster with the majority of peptides assigned to that allele was used. In cases where the majority of peptides on that cluster were assigned to a previously assigned allele (example Cluster 3 in Figure 3A) the cluster was assigned to the remaining and non-assigned allele (DRB5 ${ }^{\star} 01: 01$ allele in the previous example). After all allele-specific MCC were calculated, the median was used to compare MCCs across methods.

Bootstrapping samples of $\mathrm{N}=100$ with repetitions were used to add statistical significance to the random associations. 


\section{Sequence Logo Motifs}

All the logos in this publication were constructed from the 9-mer cores provided by each deconvolution algorithm and using Seq2Logo without clustering and using P-weighted KulblackLeibler option (23). Additionally, MHCMotifDecon web server provides sequence logos of the deconvoluted motifs using the same software with default options.

\section{Deconvolution of the DR Ligandome From Biological Samples}

To evaluate the ability of the MHCMotifDecon tool to deconvolute the DR peptidome from complex and heterozygous biological samples, we analyzed its performance on twenty seven samples from four previously published datasets, profiling the class II peptidome of cancer and autoimmune diseases. From these datasets, twelve patients with mantle cell lymphoma (MCL) (34), three patients with chronic lymphocytic leukemia (CLL) (33), five patients with rheumatoid arthritis (RA), six patients with Lyme disease (LA) (32), and one patient with sarcoidosis (35) were included in our analysis. DR peptides had been identified from clinical samples such as lymph node biopsies, splenectomy, synovial tissue, synovial fluid, PBMC and bronchoalveolar lavage (BAL) cells.

The DR peptidome for each sample along with the class II HLA type of the patient were obtained from each dataset. Since no DRB3, 4 or 5 typing had been reported, the most common secondary DRB allele with the highest LD with the reported DRB1, was assigned to each sample. Finally, the DR peptidome of each sample together with the full HLA-DR type (DRB1, 3, 4 and 5) was submitted to MHCMotifDecon using the default parameters for class II (Length range = 12-21; Class = II; Threshold for trash cluster $=20$ ) and the contribution of the DRB3, 4 and 5 molecules to the accompanying DRB1 allele was determined.

\section{Cell Lines and Antibody}

Homozygous B lymphoblastoid cell lines (BLCL) were obtained from the International Histocompatibility Working Group (IHWG) Cell and DNA bank housed at the Fred Hutchinson Cancer Research Center, Seattle, WA (http://www.ihwg.org). A group of 11 cell lines expressing the high frequency DRB3, DRB4 and DRB5 molecules along with the DRB1 alleles were selected for the study (Supplementary Table 1). To guarantee intact Class II processing and presentation machinery and to ensure that the total HLA-DR expression and the ratio of the primary and secondary DR alleles represent the physiological level use of engineered cells was avoided.

The cells were grown in high density cultures in roller bottles in complete RPMI medium (Gibco) supplemented with 15\% fetal bovine serum (FBS; Gibco/Invitrogen Corp) and 1\% $100 \mathrm{mM}$ sodium pyruvate (Gibco). Cells maintained $>90 \%$ viability at all times during the culture and were harvested from the suspension when the cell density reached to $1.5-2.5 \mathrm{e} 6$ cells $/ \mathrm{ml}$. The cells were washed twice with ice cold PBS and spun down at 2500xg at $4 \mathrm{C}$ for 10 minutes. The cell pellets were snap frozen in $\mathrm{LN}_{2}$ and stored at -80 until downstream processing.

All cell lines were subjected to high-resolution sequencebased HLA typing (HLA-A, -B, -C, DRB1,3, 4, 5, DP and DQ) immediately upon receipt and growth in our laboratory, for authentication prior to large scale culture and data collection.

The anti-human HLA-DR antibody (clone L243) was used for extraction of total HLA DR from the BLCLs. The L243 monoclonal antibody reacts with the HLA-DR antigen and does not cross react with HLA-DP and HLA-DQ. Clone L243 binds a conformational epitope on HLA-DR $\alpha$ which depends on the correct folding of the $\alpha \beta$ heterodimer and therefore can be used to purify different DR molecules regardless of the DRB chain.

\section{Isolation and Purification of HLA- DR Bound Peptides}

HLA-DR molecules were purified from homozygous cell lines by affinity chromatography using the mAb L243 (American Type Culture Collection, Manassas, VA) coupled to CNBr-activated Sepharose 4 Fast Flow (Amersham Pharmacia Biotech, Orsay, France) as described previously with some modifications (51). Briefly, frozen cell pellets were pulverized using Retsch Mixer Mill MM400, resuspended in lysis buffer comprised of Tris $\mathrm{pH}$ 8.0 (50 mM), octylphenoxy poly (ethyleneoxy) ethanol (Igepal, $0.5 \%), \mathrm{NaCl}(150 \mathrm{mM})$ and complete protease inhibitor cocktail (Roche, Mannheim, Germany). Lysates were centrifuged at $200,000 \mathrm{xg}$ for $90 \mathrm{~min}$ in an Optima XPN-80 ultracentrifuge (Beckman Coulter, IN, USA) and filtered supernatants were loaded on immunoaffinity columns. After a minimum of 3 passages, columns were washed sequentially with a series of wash buffers (51) and were eluted with $0.2 \mathrm{~N}$ acetic acid. The HLA was denatured, and the peptides were isolated by adding glacial acetic acid (up to 10\%) and heat. The mixture of Peptides and HLA-DR was subjected to reverse phase high performance liquid chromatography (RP-HPLC).

\section{Fractionation of the HLA/Peptide Mixture by RP-HPLC}

Reverse-phase high performance liquid chromatography (RPHPLC) was used to reduce the complexity of the peptide mixture eluted from the affinity column. First, the eluate was dried under vacuum using a CentriVap concentrator (Labconco, Kansas City, Missouri, USA). The solid residue was dissolved in $10 \%$ acetic acid in water and fractionated over a $150-\mathrm{mm}$ long Gemini $\mathrm{C}_{18}$ column, pore size $110 \AA$, particle size $5 \mu$ (Phenomenex, Torrance, California, USA) using a Paradigm MG4 instrument (Michrom BioResources, Auburn, California, USA). An acetonitrile (ACN) gradient was run at $\mathrm{pH} 2$ using a two-solvent system. Solvent A contained $2 \% \mathrm{ACN}$ in water, and solvent B contained 5\% water in ACN. Both solvent A and Solvent $B$ contained $0.1 \%$ trifluoroacetic acid (TFA). The column was preequilibrated at $2 \%$ solvent $\mathrm{B}$. The sample was loaded on the column in a period of 18 minutes using a solvent system comprised of $2 \%$ solvent $B$ at a flow rate of $120 \mu \mathrm{l} / \mathrm{min}$. Then two linear gradients were run at $160 \mu \mathrm{l} / \mathrm{min}$ flow rate: $4 \%$ to $40 \%$ Solvent B for $40 \mathrm{~min}$, followed by $40 \%$ to $80 \%$ Solvent B for $8 \mathrm{~min}$.

The percentage of Solvent B was maintained at $80 \%$ for $4 \mathrm{~min}$, and then decreased to $2 \%$ over a period of $3 \mathrm{~min}$. Fractions were collected in 2 min intervals using a Gilson FC 203B fraction 
collector (Gilson, Middleton, Wisconsin, USA), and the ultraviolet (UV) absorption profile of the eluate was recorded at 215 nm wavelength.

\section{Nano LC-MS/MS Analysis}

Peptide-containing HPLC fractions were dried, resuspended in an aqueous solvent composed of $10 \%$ acetic acid, $2 \% \mathrm{ACN}$ and iRT peptides (Biognosys, Schlieren, Switzerland) as internal standards. Fractions were applied individually to an Eksigent nanoLC 415 nanoscale RP-HPLC (AB Sciex, Framingham, Massachusetts, USA), including a 5-mm long, $350 \mu \mathrm{m}$ internal diameter Chrom XP C18 trap column with $3-\mu \mathrm{m}$ particles and $120 \AA$ pores, and a $15-\mathrm{cm}$-long ChromXP C18 separation column (75- $\mu \mathrm{m}$ internal diameter) packed with the same medium (AB Sciex, Framingham, Massachusetts, USA). An ACN gradient was run at pH 2.5 using a two-solvent system. Solvent A was $0.1 \%$ formic acid in water, and solvent B was $0.1 \%$ formic acid in $95 \%$ $\mathrm{ACN}$ in water. The column was pre-equilibrated at $2 \%$ solvent B. Samples were loaded at $5 \mu \mathrm{L} / \mathrm{min}$ flow rate onto the trap column and run through the separation column at 300 $\mathrm{nL} / \mathrm{min}$ with two linear gradients: $10 \%$ to $40 \% \mathrm{~B}$ for 70 minutes, followed by $40 \%$ to $80 \%$ B for 7 minutes. The column effluent was next injected using the nanospray III ion source of an $A B$ Sciex TripleTOF 5600 quadrupole time-of-flight mass spectrometer (AB Sciex, Framingham, MA, USA) with the source voltage set to $2,400 \mathrm{v}$. Information-dependent analysis (IDA) of peptide ions was acquired based on a survey scan in the TOF-MS positive-ion mode over a range of 300 to $1,250 \mathrm{~m} / \mathrm{z}$ for 0.25 seconds. Following each survey scan, up to 22 ions with a charge state of 2 to 5 and intensity of at least 200 counts per second were subjected to collision-induced dissociation (CID) for tandem MS analysis (MS/MS) over a maximum period of 3.3 seconds. Selection of a particular ion $\mathrm{m} / \mathrm{z}$ was excluded for 30 seconds after three initial MS/MS experiments. Dynamic collision energy was utilized to automatically adjust the collision voltage based upon ion size and charge. PeakView Software version 1.2.0.3 (AB Sciex, Framingham, MA, USA) was used for data visualization.

\section{Data Analysis}

Peptide sequences were identified using PEAKS Studio 10.5 software (Bioinformatics Solutions, Waterloo, Canada) at a precursor mass error tolerance of $30 \mathrm{ppm}$ and a fragment mass error tolerance of $0.02 \mathrm{Da}$. A database composed of SwissProt Homo sapiens (taxon identifier 9606) and iRT peptide sequences was used as the reference for database search. Variable posttranslational modifications (PTM) including acetylation, deamination, pyroglutamate formation, oxidation, sodium adducts, phosphorylation, and cysteinylation were included in database search. Identified peptides were further filtered at a false discovery rate (FDR) of $1 \%$ using PEAKS decoyfusion algorithm.

\section{Peptide Repertoire Overlap Analysis}

To determine the degree of overlap between the peptide repertoire of the primary and secondary DR molecule in each haplotype, the total DR peptide repertoire of each cell line was submitted to NetMHCIIpan-4.1 and the predicted binding to the primary and secondary molecule was calculated. Peptide binders were defined using an EL rank threshold of 1\%, peptides that showed binding to both molecules were considered overlapping. Note, that this analysis was performed using a different predicted binding threshold (1\%) compared to that used by MHCMotifDecon (20\%). This is because the latter analysis was performed to exclude the co-purified contaminants, while the former was done to compare the overlap between highly reliable peptide repertoires. Therefore, the reported relative repertoire sizes of the primary and secondary DR molecule can be slightly different between the two analyses.

\section{DATA AVAILABILITY STATEMENT}

The raw data supporting the conclusions of this article will be made available by the authors, without undue reservation. The HLA-DR immunopeptidome data set from the 11 cell lines used in the study is available in Supplementary Table 4.

\section{AUTHOR CONTRIBUTIONS}

Conceptualization, SK, CB, WH, and MN. Methodology, SK, CB, $\mathrm{BA}$, and $\mathrm{MN}$. Investigation, $\mathrm{SK}, \mathrm{CB}, \mathrm{BA}, \mathrm{HY}$, and MN. Writing Original Draft, SK, CB, HY, WH, and MN. Writing - Review \& Editing, SK, CB, and MN. Funding Acquisition and Resources, $\mathrm{WH}$ and MN. Supervision, SK, CB, and MN. All authors contributed to the article and approved the submitted version.

\section{FUNDING}

This research was funded in part through US Federal funds from the National Institute of Allergy and Infectious Diseases, National Institutes of Health and Department of Health and Human Services (under Contract No. HHSN272201200010CERC to MN).

\section{ACKNOWLEDGMENTS}

We would like to thank Dr. Rico Buchli (Pure Protein, LLC) for providing the L243 affinity columns for this study. We sincerely thank Steven Cate (University of Oklahoma Health Sciences Center) and Sean Osborn (Pure MHC, LLC) for HLA typing of the BLCLs and very helpful discussions.

\section{SUPPLEMENTARY MATERIAL}

The Supplementary Material for this article can be found online at: https://www.frontiersin.org/articles/10.3389/fimmu.2022. 835454/full\#supplementary-material 


\section{REFERENCES}

1. Barra C, Ackaert C, Reynisson B, Schockaert J, Jessen LE, Watson M, et al. Immunopeptidomic Data Integration to Artificial Neural Networks Enhances Protein-Drug Immunogenicity Prediction. Front Immunol (2020) 11:1304. doi: $10.3389 /$ fimmu.2020.01304

2. Bettencourt P, Müller J, Nicastri A, Cantillon D, Madhavan M, Charles PD, et al. Identification of Antigens Presented by MHC for Vaccines Against Tuberculosis. NPJ Vaccines (2020) 5(1):2. doi: 10.1038/s41541-019-0148-y

3. Mayer RL, Impens F. Immunopeptidomics for Next-Generation Bacterial Vaccine Development. Trends Microbiol (2021) 29(11):1034-45. doi: 10.1016/ j.tim.2021.04.010

4. Nelde A, Rammensee HG, Walz JS. The Peptide Vaccine of the Future. Mol Cell Proteomics (2021) 20:100022. doi: 10.1074/mcp.R120.002309

5. Abelin JG, Keskin DB, Sarkizova S, Hartigan CR, Zhang W, Sidney J, et al. Mass Spectrometry Profiling of HLA-Associated Peptidomes in Mono-Allelic Cells Enables More Accurate Epitope Prediction. Immunity (2017) 46(2):31526. doi: 10.1016/j.immuni.2017.02.007

6. Sarkizova S, Klaeger S, Le PM, Li LW, Oliveira G, Keshishian H, et al. A Large Peptidome Dataset Improves HLA Class I Epitope Prediction Across Most of the Human Population. Nat Biotechnol (2020) 38(2):199-209. doi: 10.1038/ s41587-019-0322-9

7. Abelin JG, Harjanto D, Malloy M, Suri P, Colson T, Goulding SP, et al. Defining HLA-II Ligand Processing and Binding Rules With Mass Spectrometry Enhances Cancer Epitope Prediction. Immunity (2019) 51 (4):766-79.e17. doi: 10.1016/j.immuni.2019.08.012

8. Andreatta M, Alvarez B, Nielsen M. GibbsCluster: Unsupervised Clustering and Alignment of Peptide Sequences. Nucleic Acids Res (2017) 45(W1): W458-w63. doi: 10.1093/nar/gkx248

9. Andreatta M, Lund O, Nielsen M. Simultaneous Alignment and Clustering of Peptide Data Using a Gibbs Sampling Approach. Bioinformatics (2013) 29 (1):8-14. doi: 10.1093/bioinformatics/bts621

10. Racle J, Michaux J, Rockinger GA, Arnaud M, Bobisse S, Chong C, et al. Robust Prediction of HLA Class II Epitopes by Deep Motif Deconvolution of Immunopeptidomes. Nat Biotechnol (2019) 37(11):1283-6. doi: 10.1038/ s41587-019-0289-6

11. Alvarez B, Barra C, Nielsen M, Andreatta M. Computational Tools for the Identification and Interpretation of Sequence Motifs in Immunopeptidomes. Proteomics (2018) 18(12):e1700252. doi: 10.1002/pmic.201700252

12. Mommen GP, Marino F, Meiring HD, Poelen MC, van Gaans-van den Brink JA, Mohammed S, et al. Sampling From the Proteome to the Human Leukocyte Antigen-DR (HLA-DR) Ligandome Proceeds Via High Specificity. Mol Cell Proteomics (2016) 15(4):1412-23. doi: 10.1074/ mcp.M115.055780

13. Parker R, Partridge T, Wormald C, Kawahara R, Stalls V, Aggelakopoulou M, et al. Mapping the SARS-CoV-2 Spike Glycoprotein-Derived Peptidome Presented by HLA Class II on Dendritic Cells. Cell Rep (2021) 35 (8):109179. doi: 10.1016/j.celrep.2021.109179

14. Sofron A, Ritz D, Neri D, Fugmann T. High-Resolution Analysis of the Murine MHC Class II Immunopeptidome. Eur J Immunol (2016) 46(2):31928. doi: 10.1002/eji.201545930

15. Alvarez B, Reynisson B, Barra C, Buus S, Ternette N, Connelley T, et al. NNAlign_MA; MHC Peptidome Deconvolution for Accurate MHC Binding Motif Characterization and Improved T-Cell Epitope Predictions. Mol Cell Proteomics (2019) 18(12):2459-77. doi: 10.1074/mcp.TIR119.001658

16. Reynisson B, Alvarez B, Paul S, Peters B, Nielsen M. NetMHCpan-4.1 and NetMHCIIpan-4.0: Improved Predictions of MHC Antigen Presentation by Concurrent Motif Deconvolution and Integration of MS MHC Eluted Ligand Data. Nucleic Acids Res (2020) 48(W1):W449-w54. doi: 10.1093/nar/gkaa379

17. Apps R, Meng Z, Del Prete GQ, Lifson JD, Zhou M, Carrington M. Relative Expression Levels of the HLA Class-I Proteins in Normal and HIV-Infected Cells. J Immunol (2015) 194(8):3594-600. doi: 10.4049/jimmunol.1403234

18. Bassani-Sternberg M, Gfeller D. Unsupervised HLA Peptidome Deconvolution Improves Ligand Prediction Accuracy and Predicts Cooperative Effects in Peptide-HLA Interactions. J Immunol (2016) 197 (6):2492-9. doi: 10.4049/jimmunol.1600808

19. Partridge T, Nicastri A, Kliszczak AE, Yindom LM, Kessler BM, Ternette N, et al. Discrimination Between Human Leukocyte Antigen Class I-Bound and
Co-Purified HIV-Derived Peptides in Immunopeptidomics Workflows. Front Immunol (2018) 9:912. doi: 10.3389/fimmu.2018.00912

20. Ben Dror L, Barnea E, Beer I, Mann M, Admon A. The HLA-B ${ }^{\star} 2705$ Peptidome. Arthritis Rheum (2010) 62(2):420-9. doi: 10.1002/art.27257

21. Trolle T, McMurtrey CP, Sidney J, Bardet W, Osborn SC, Kaever T, et al. The Length Distribution of Class I-Restricted T Cell Epitopes Is Determined by Both Peptide Supply and MHC Allele-Specific Binding Preference. J Immunol (2016) 196(4):1480-7. doi: 10.4049/jimmunol.1501721

22. Fisch A, Reynisson B, Benedictus L, Nicastri A, Vasoya D, Morrison I, et al. Integral Use of Immunopeptidomics and Immunoinformatics for the Characterization of Antigen Presentation and Rational Identification of BoLA-DR-Presented Peptides and Epitopes. J Immunol (2021) 206 (10):2489-97. doi: 10.4049/jimmunol.2001409

23. Thomsen MC, Nielsen M. Seq2Logo: A Method for Construction and Visualization of Amino Acid Binding Motifs and Sequence Profiles Including Sequence Weighting, Pseudo Counts and Two-Sided Representation of Amino Acid Enrichment and Depletion. Nucleic Acids Res (2012) 40(Web Server issue):W281-7. doi: 10.1093/nar/gks469

24. Li Y, Li H, Martin R, Mariuzza RA. Structural Basis for the Binding of an Immunodominant Peptide From Myelin Basic Protein in Different Registers by Two HLA-DR2 Proteins. J Mol Biol (2000) 304(2):177-88. doi: 10.1006/ jmbi.2000.4198

25. Reynisson B, Barra C, Kaabinejadian S, Hildebrand WH, Peters B, Nielsen M. Improved Prediction of MHC II Antigen Presentation Through Integration and Motif Deconvolution of Mass Spectrometry MHC Eluted Ligand Data. J Proteome Res (2020) 19(6):2304-15. doi: 10.1021/acs.jproteome.9b00874

26. Scholz EM, Marcilla M, Daura X, Arribas-Layton D, James EA, Alvarez I. Human Leukocyte Antigen (HLA)-DRB1*15:01 and HLA-DRB5*01:01 Present Complementary Peptide Repertoires. Front Immunol (2017) 8:984. doi: 10.3389/fimmu.2017.00984

27. Smith KJ, Pyrdol J, Gauthier L, Wiley DC, Wucherpfennig KW. Crystal Structure of HLA-DR2 $\left(\mathrm{DRA}^{\star} 0101, \mathrm{DRB}^{\star}{ }^{1501}\right)$ Complexed With a Peptide From Human Myelin Basic Protein. J Exp Med (1998) 188(8):1511-20. doi: 10.1084/jem.188.8.1511

28. Vogt AB, Kropshofer H, Kalbacher H, Kalbus M, Rammensee HG, Coligan JE, et al. Ligand Motifs of HLA-DRB5 ${ }^{\star} 0101$ and DRB1*1501 Molecules Delineated From Self-Peptides. J Immunol (1994) 153(4):1665-73.

29. Voorter CE, Lardy NM, van den Berg-Loonen EM. Presence of the DRB4*0103102N Null Allele in Different DRB1*04-Positive Individuals. Tissue Antigens (2000) 55(1):37-43. doi: 10.1034/j.1399-0039.2000.550106.x

30. Parry CS, Gorski J, Stern LJ. Crystallographic Structure of the Human Leukocyte Antigen DRA, DRB3 ${ }^{\star} 0101$ : Models of a Directional Alloimmune Response and Autoimmunity. J Mol Biol (2007) 371(2):435-46. doi: 10.1016/ j.jmb.2007.05.025

31. Dai S, Crawford F, Marrack P, Kappler JW. The Structure of HLA-DR52c: Comparison to Other HLA-DRB3 Alleles. Proc Natl Acad Sci USA (2008) 105 (33):11893-7. doi: 10.1073/pnas.0805810105

32. Wang Q, Drouin EE, Yao C, Zhang J, Huang Y, Leon DR, et al. Immunogenic HLA-DR-Presented Self-Peptides Identified Directly From Clinical Samples of Synovial Tissue, Synovial Fluid, or Peripheral Blood in Patients With Rheumatoid Arthritis or Lyme Arthritis. J Proteome Res (2017) 16(1):122-36. doi: 10.1021/acs.jproteome.6b00386

33. Nelde A, Kowalewski DJ, Backert L, Schuster H, Werner JO, Klein R, et al. HLA Ligandome Analysis of Primary Chronic Lymphocytic Leukemia (CLL) Cells Under Lenalidomide Treatment Confirms the Suitability of Lenalidomide for Combination With T-Cell-Based Immunotherapy. Oncoimmunology (2018) 7(4):e1316438. doi: 10.1080/2162402X.2017. 1316438

34. Khodadoust MS, Olsson N, Wagar LE, Haabeth OA, Chen B, Swaminathan K, et al. Antigen Presentation Profiling Reveals Recognition of Lymphoma Immunoglobulin Neoantigens. Nature (2017) 543(7647):723-7. doi: 10.1038 /nature21433

35. Heyder T, Kohler M, Tarasova NK, Haag S, Rutishauser D, Rivera NV, et al. Approach for Identifying Human Leukocyte Antigen (HLA)-DR Bound Peptides From Scarce Clinical Samples. Mol Cell Proteomics (2016) 15 (9):3017-29. doi: 10.1074/mcp.M116.060764

36. Caillier SJ, Briggs F, Cree BA, Baranzini SE, Fernandez-Viña M, Ramsay PP, et al. Uncoupling the Roles of HLA-DRB1 and HLA-DRB5 Genes in Multiple 
Sclerosis. J Immunol (2008) 181(8):5473-80. doi: 10.4049/jimmunol. 181.8.5473

37. Faner R, James E, Huston L, Pujol-Borrel R, Kwok WW, Juan M. Reassessing the Role of HLA-DRB3 T-Cell Responses: Evidence for Significant Expression and Complementary Antigen Presentation. Eur J Immunol (2010) 40(1):91102. doi: $10.1002 /$ eji.200939225

38. Prat E, Tomaru U, Sabater L, Park DM, Granger R, Kruse N, et al. HLADRB ${ }^{*} 0101$ and $-\mathrm{DRB1}^{*} 1501$ Expression in the Multiple Sclerosis-Associated HLA-DR15 Haplotype. J Neuroimmunol (2005) 167(1-2):108-19. doi: 10.1016/j.jneuroim.2005.04.027

39. Leën MP, Gorski J. DRB4 Promoter Polymorphism in DR7 Individuals: Correlation With DRB4 pre-mRNA and mRNA Levels. Immunogenetics (1997) 45(6):371-8. doi: 10.1007/s002510050218

40. Stunz LL, Karr RW, Anderson RA. HLA-DRB1 and -DRB4 Genes Are Differentially Regulated at the Transcriptional Level. J Immunol (1989) 143 (9):3081-6

41. Bettencourt A, Carvalho C, Leal B, Brás S, Lopes D, Martins da Silva A, et al. The Protective Role of HLA-DRB1(*)13 in Autoimmune Diseases. J Immunol Res (2015) 2015:948723. doi: 10.1155/2015/948723

42. Furukawa H, Kawasaki A, Oka S, Ito I, Shimada K, Sugii S, et al. Human Leukocyte Antigens and Systemic Lupus Erythematosus: A Protective Role for the HLA-DR6 Alleles DRB1¹3:02 and ${ }^{*}$ 14:03. PloS One (2014) 9(2):e87792. doi: 10.1371/journal.pone.0087792

43. Furukawa H, Oka S, Tsuchiya N, Shimada K, Hashimoto A, Tohma S, et al. The Role of Common Protective Alleles HLA-DRB1 ${ }^{\star} 13$ Among Systemic Autoimmune Diseases. Genes Immun (2017) 18(1):1-7. doi: 10.1038/ gene.2016.40

44. Arango MT, Perricone C, Kivity S, Cipriano E, Ceccarelli F, Valesini G, et al. HLA-DRB1 the Notorious Gene in the Mosaic of Autoimmunity. Immunol Res (2017) 65(1):82-98. doi: 10.1007/s12026-016-8817-7

45. Texier C, Pouvelle-Moratille S, Busson M, Charron D, Ménez A, Maillère B. Complementarity and Redundancy of the Binding Specificity of HLA-DRB1, -DRB3, -DRB4 and -DRB5 Molecules. Eur J Immunol (2001) 31(6):1837-46. doi: 10.1002/1521-4141(200106)31:6<1837::AID-IMMU1837>3.0.CO;2-H

46. Wucherpfennig KW, Sette A, Southwood S, Oseroff C, Matsui M, Strominger JL, et al. Structural Requirements for Binding of an Immunodominant Myelin Basic Protein Peptide to DR2 Isotypes and for its Recognition by Human T Cell Clones. J Exp Med (1994) 179(1):279-90. doi: 10.1084/jem.179.1.279
47. Gupta S, Höpner S, Rupp B, Günther S, Dickhaut K, Agarwal N, et al. Anchor Side Chains of Short Peptide Fragments Trigger Ligand-Exchange of Class II MHC Molecules. PloS One (2008) 3(3):e1814. doi: 10.1371/journal.pone.0001814

48. Hammer J, Gallazzi F, Bono E, Karr RW, Guenot J, Valsasnini P, et al. Peptide Binding Specificity of HLA-DR4 Molecules: Correlation With Rheumatoid Arthritis Association. J Exp Med (1995) 181(5):1847-55. doi: 10.1084/ jem.181.5.1847

49. James EA, Gillette L, Durinovic-Bello I, Speake C, Bondinas GP, Moustakas AK, et al. DRB $4^{*} 01: 01$ Has a Distinct Motif and Presents a Proinsulin Epitope That Is Recognized in Subjects With Type 1 Diabetes. J Immunol (2018) 201 (12):3524-33. doi: 10.4049/jimmunol.1800723

50. Wang J, Jelcic I, Mühlenbruch L, Haunerdinger V, Toussaint NC, Zhao Y, et al. HLA-DR15 Molecules Jointly Shape an Autoreactive T Cell Repertoire in Multiple Sclerosis. Cell (2020) 183(5):1264-81.e20. doi: 10.1016/j.cell. 2020.09.054

51. Purcell AW, Ramarathinam SH, Ternette N. Mass Spectrometry-Based Identification of MHC-Bound Peptides for Immunopeptidomics. Nat Protoc (2019) 14(6):1687-707. doi: 10.1038/s41596-019-0133-y

Conflict of Interest: SK is an employee at Pure MHC, LLC.

The remaining authors declare that the research was conducted in the absence of any commercial or financial relationships that could be construed as a potential conflict of interest.

Publisher's Note: All claims expressed in this article are solely those of the authors and do not necessarily represent those of their affiliated organizations, or those of the publisher, the editors and the reviewers. Any product that may be evaluated in this article, or claim that may be made by its manufacturer, is not guaranteed or endorsed by the publisher.

Copyright $\odot 2022$ Kaabinejadian, Barra, Alvarez, Yari, Hildebrand and Nielsen. This is an open-access article distributed under the terms of the Creative Commons Attribution License (CC BY). The use, distribution or reproduction in other forums is permitted, provided the original author(s) and the copyright owner(s) are credited and that the original publication in this journal is cited, in accordance with accepted academic practice. No use, distribution or reproduction is permitted which does not comply with these terms. 